\title{
Hans Kelsens Vorschläge zur Reform der österreichisch-ungarischen Wehrverfassung
}

\begin{abstract}
Hans Kelsen's proposals for a reform of the Austro-Hungarian military organization.
In 1917 Hans Kelsen became legal advisor to the last Austro-Hungarian minister of war, Rudolf Stöger-Steiner. In this position Kelsen wrote several reports on his ideas to reform the Austro-Hungarian monarchy - including two opinions on the reorganization of the Austro-Hungarian armed forces from a legal point of view. Kelsen suggested to divide the whole army in an Austrian and a Hungarian part but to retain a common ministry of war for both parts of the monarchy.
\end{abstract}

Keywords: Army reform - Austro-Hungarian Army - Dualism - Hans KELSEN - Rudolf STÖGER-STEINER

\section{Einleitung}

Ein Krieg wie der Erste Weltkrieg brachte fast schon zwangsläufig eine Konjunktur des Militärrechts mit sich, und so wurde 1917 in Österreich eine neue Zeitschrift gegründet, die sich speziell mit diesem Rechtsgebiet befassen sollte. Die meisten Artikel der neuen "Zeitschrift für Militärrecht" beschäftigten sich mit Fragen des Militärstrafrechts, der erste Artikel des ersten Hefts aber enthielt Hans Kelsens Gedanken "Zur Reform der verfassungsrechtlichen Grundlagen der Wehrmacht Österreich-Ungarns". ${ }^{1}$

Dieser Artikel führte dazu, dass Kelsen als juristischer Berater von Minister Rudolf Freiherrr Stöger-Steiner von Steinstätten in das gemeinsame Kriegsministerium der österreichischungarischen Monarchie berufen wurde. ${ }^{2}$ Wäh-

\footnotetext{
${ }^{1}$ KELSEN, Reform.

2 Dazu: MÉTALl, Kelsen 19-28; Busch, Kelsen im Ersten Weltkrieg 67-70; OBERKOFLER, RABOFSKY, Kelsen im Kriegseinsatz.
}

rend seiner Tätigkeit für Stöger-Steiner ${ }^{3}$ beschäftigte sich Kelsen weiterhin mit Reformüberlegungen; er machte allgemeine Vorschläge für die Reform der Monarchie und spezifische Vorschläge für die Reform der Armee, genauer für die staatsrechtliche Stellung der Armee im Gefüge der Doppelmonarchie. $\mathrm{Zu}$ diesem Thema liegen von Kelsen zwei Gutachten vor, ${ }^{4}$ wobei einem Gutachten ein Entwurf für ein Militärgesetz angeschlossen ist. ${ }^{5}$ Im Folgenden werden diese beiden Gutachten zu militärischen Fragen eingeleitet und ediert.

\footnotetext{
${ }^{3} \mathrm{Zu}$ Stöger-Steiner: SCHMIDT-BRENTANO, StögerSteiner; STEINER, Österreich-Ungarns letzter Kriegsminister.

${ }^{4}$ Die Gutachten befinden sich in der Handschriftensammlung der Österreichischen Nationalbibliothek: Cod. Ser. n. 54574 (Reform der Organisation der Wehrmacht)

Cod. Ser. n. 54580 (Die Aufgaben der Heeresverwaltung bei einer Verfassungsreform)

${ }^{5}$ Dieses Gutachten wurde bereits ediert bei: ОвеRKOFLER, RABOFSKY, Kelsen im Kriegseinsatz 174-186 (Dokument III), allerdings ohne jene Passagen, die im ursprünglichen Texte enthalten waren, dann aber gestrichen wurden.
} 


\section{Die Ausgangssituation}

In seinen militärrechtlichen Gutachten für Stöger-Steiner beschäftigte sich Kelsen vor allem mit zwei Fragen, nämlich einerseits mit der Position des Militärs im dualistischen System und andererseits mit der Abgrenzung von Kommandogewalt und Militärverwaltung. Außerdem widmete er sich der Frage, wie die militärischen Interessen im Rahmen einer Verfassungsreform berücksichtigt werden konnten.

\section{Das Militär im System des Ausgleichs}

Das Militärwesen hatte 1867/68 jene rechtliche Struktur bekommen, die es bis zum Ende der Monarchie behalten sollte. ${ }^{6}$ Diese Struktur war vergleichsweise komplex. An sich war das Kriegswesen eine pragmatisch-gemeinsame Angelegenheit beider Reichshälften. ${ }^{7}$ Dafür war eines der drei gemeinsamen Ministerien zuständig, das gemeinsame Kriegsministerium, das bis 1911 auch als Reichskriegsministerium bezeichnet wurde. ${ }^{8}$

Gewisse militärische Bereiche - insbesondere die Feststellung des Wehrsystems - waren aber dualistische Angelegenheiten. ${ }^{9}$ Für sie waren also keine gemeinsamen Behörden zuständig, sondern die beiden Rechtshälften, die jene Bereiche aber nach gemeinsamen Grundsätzen regeln sollten. Schließlich gab es noch einzelne militärische Angelegenheiten, die von den beiden Reichshälften autonom geregelt werden konnten. ${ }^{10}$ Für die dualistischen und autonomen Angelegenheiten waren Behörden der beiden

\footnotetext{
${ }^{6}$ OlechowsKI-HrdlickA, Gemeinsame Angelegenheiten 265-290 mit zahlreichen weiteren Nachweisen; SCHMID, Heerwesen 737-738; Ivan ZOLGER, Staatsrechtliche Grundlagen.

7 § 1 lit. b DG, RGBl. 145/1867.

${ }^{8}$ WAGNER, k.(u.)k. Armee 351-372.

${ }^{9} \S 2$ Z. 5 DG.

${ }^{10}$ Umkehrschluss aus $\S 1$ lit. b DG; SCHMID, Heerwesen 738 .
}

Reichshälften zuständig und keine gemeinsamen. Sowohl in Wien als auch in Budapest wurde ein Landesverteidigungsministerium zur Verwaltung dieser Angelegenheiten eingerichtet. Somit gab es nach dem Ausgleich in der Monarchie drei Ministerien für den militärischen Bereich: das gemeinsame k.(u.)k. Kriegsministerium, das österreichische (k.k.) Kriegsministerium und das ungarische Landesverteidigungsministerium. ${ }^{11}$

Nach den Militärbehörden wurden 1868 auch die Truppen selbst dreigeteilt. Das Ideal der national-ungarischen Politiker wäre schon beim Ausgleich eine Teilung der bestehenden Armee in einen österreichischen und einen ungarischen Teil gewesen. Franz Joseph beharrte aber auf einer gemeinsamen Armee. Als Kompromiss wurde zusätzlich zur gemeinsamen Armee beider Reichshälften eine ungarische Landwehr (Honved) geschaffen, um den ungarischen Wunsch nach einer eigenen Armee zu erfüllen. In der österreichischen Reichshälfte gab es kein vergleichsbares Bedürfnis nach eigenen Truppen neben der gemeinsamen Armee; trotzdem erhielt auch die österreichische Reichshälfte aus Gründen der Parität die k.k. Landwehr. Somit gab es in der Habsburgermonarchie auch drei Armeen: die gemeinsame k.(u.)k. Armee, die österreichische (k.k.) Landwehr und die ungarische Honved. ${ }^{12}$

\footnotetext{
${ }^{11}$ Zum gemeinsamen Kriegsministerium: WAGNER, Kriegsministeriums; DeRS., k.(u.)k. Armee 351-372; zum österreichischen Landesverteidigungsministerium: WAGNER, k.(u.)k. Armee 417-418; zum ungarischen Landesverteidigungsministerium: PAPP, Honved 645-646.

12 Allmayer-BeCK, Bewaffnete Macht 81-87; SCHMID, Heeresrecht 26-29

Nach der Okkupation Bosnien-Herzegowinas erhielt die Bewaffnete Macht der Habsburgermonarchie sogar noch einen vierten Teil, nämlich die bosnischherzegowinischen Truppen. Diese waren insofern gemeinsam, als Bosnien-Herzegowina von beiden Reichshälften gemeinsam (und zwar durch den gemeinsamen Finanzminister) verwaltet wurde, gleich-
} 
Die beiden Landwehren waren eigenartige Konstruktionen, die nicht aus militärischen, sondern aus politischen Erwägungen geschaffen wurden. Daher unterschieden sich die beiden Landwehren der Habsburgermonarchie wesentlich von der Landwehr im Deutschen Kaiserreich. Dort bestand die Landwehr aus Truppen Zweiter Linie, also einer Art Reserve, während sich die Landwehren in Österreich-Ungarn immer mehr zu vollwertigen, regulären Truppen entwickelten. Die Landwehren wurden gleichzeitig aber so gestaltet, dass sie selbständig, ohne die gemeinsame Armee nicht einsatzfähig waren. Die Landwehren bestanden (bis nach der Jahrhundertwende) lediglich aus Infanterie und Kavallerie. Sie hatten weder Artillerie noch technische Truppen und auch keine höheren Kommandostrukturen. Der Kaiser wollte nämlich unbedingt vermeiden, dass die ungarische Honved in einem Bürgerkrieg eigenständig gegen den Rest der Monarchie kämpfen konnte. ${ }^{13}$

Der Kompromiss von einer gemeinsamen Armee und zwei Landwehren befriedigte die Nationalisten in Ungarn nicht dauerhaft. Gerade in den Jahren nach 1900 kamen wieder ungarische Forderungen nach einer Teilung auch der gemeinsamen Armee - oder zumindest mehr ungarischer Eigenständigkeit im militärischen Bereich, die schließlich im sogenannten „Neunerprogramm“ zusammengefasst wurden. ${ }^{14}$ Diese Forderungen stützten sich auf eine nicht ganz eindeutige Formulierung des ungarischen Aus-

zeitig waren sie aber kein Teil der gemeinsamen Armee und trugen auch nicht die Bezeichnung ,k.u.k“. 13 Allmayer-BeCK, Bewaffnete Macht 81-87, SCHMID, Heeresrecht 27.

${ }^{14}$ Das Neunerprogramm wurde von einem neunköpfigen Komitee der liberalen Partei ausgearbeitet und war dann die militärpolitische Grundlage der ersten Regierung von Istvan Tisza von 1903 bis 1905. Eine deutsche Übersetzung ist abgedruckt bei: BERNATZIK, Verfassungsgesetze Nr.178d; zum Hintergrund siehe auch FRECH, Armeefrage; KOLMER, Parlament und Verfassung 507-508. gleichsgesetzes. In dessen $\S 11$ wurde das „ungarische Heer" als „ergänzender Teil der Gesamtarmee" bezeichnet. ${ }^{15}$ Ungarische Nationalisten betonten (in einer strikten Wortinterpretation), dass hier von einem (eigenen) „ungarischen Heer" die Rede war. Für Kaiser Franz Joseph war eine Teilung der gemeinsamen Armee weiterhin undenkbar. Als Reaktion auf die ungarischen Teilungsbestrebungen erließ er 1903 nach Manövern im galizischen Chlopy einen Armeebefehl, in dem er erklärte:

„Mein Heer insbesondere [...] möge wissen, daß ich nie der Rechte und Befugnisse mich begebe, welche seinem Obersten Herrn verbürgt sind. Gemeinsam und einheitlich, wie es ist, soll Mein Heer bleiben, die starke Macht zur Vertheidigung der österreichisch-ungarischen Monarchie gegen jeden Feind. ${ }^{\prime 16}$

Obwohl Franz Joseph strikt an der Gemeinsamkeit der Armee festhielt, erfüllte er nach der Jahrhundertwende mehrere ungarische Forderungen aus dem Neunerprogramm. ${ }^{17}$ Außerdem erhielt die Honved (und daher auch die k.k. Landwehr) nun doch eigene Artillerieeinheiten. ${ }^{18}$

\section{Kommandogewalt und Militärverwaltung}

Im konstitutionellen System wurde bei der Führung der Armee grundsätzlich zwischen Kommandogewalt einerseits und Militärverwaltung andererseits unterschieden. Der Oberbefehl war

\footnotetext{
${ }^{15}$ GA XII:1867, abgedruckt bei BERNATZIK, Verfassungsgesetze Nr. 119.

16 PÉTER, Verfassungsentwicklung in Ungarn 527.

${ }_{17}$ BERNATZIK, Verfassungsgesetze 706f; auch der 1911

- beim Erscheinen der zweiten Auflage von Bernatziks Buch - noch nicht erfüllte Punkt 2 (neue Militärstrafprozessordnung mit mündlicher Verhandlung in ungarischer Sprache für ungarische Staatsbürger) wurde schließlich durch die MilStPO 1912 (RGBl. 130/1912) erfüllt.

18 PAPP, Honved 657-658; WAGNER, k.(u.)k. Armee $427-428$.
} 
eine Prärogative (auch Reservatrecht genannt) des Monarchen. ${ }^{19}$ Das bedeutete, dass Anordnungen des Kaisers nicht von einem Minister gegengezeichnet werden mussten, um gültig zu sein. In Bezug auf die Kommandogewalt gab es also keine Ministerverantwortlichkeit. In der Militärverwaltung hingegen sollte das System von Gegenzeichnung und Ministerverantwortlichkeit (zumindest in der Theorie) genauso wie in anderen Bereichen der Verwaltung gelten. ${ }^{20}$

In der Habsburgermonarchie waren die militärischen Prärogativen des Monarchen nach 1867 im positiven Recht verankert. $\S 5$ des österreichischen Delegationsgesetzes legte zur Verwaltung der pragmatisch-gemeinsamen Angelegenheiten grundsätzlich fest:

„Die Verwaltung der gemeinsamen Angelegenheiten wird durch ein gemeinsames verantwortliches Ministerium besorgt, welchem jedoch nicht gestattet ist, nebst den gemeinsamen Angelegenheiten auch die besonderen Regierungsgeschäfte einer der beiden Reichstheile zu führen. ${ }^{21}$

Die Verantwortlichkeit der gemeinsamen Minister war freilich eine theoretische, weil die Ausführungsgesetze über das genaue Verfahren dazu nie erlassen wurden.22 Für den militärischen Bereich wurde außerdem im 2. Absatz des $\S 5$ Delegationsgesetz eine Ausnahme normiert: „Die Anordnungen in Betreff auf Leitung, Führung und inneren Organisation der gesammten Armee stehen ausschließlich dem Kaiser zu. ${ }^{{ }^{\prime 2} 3}$

\footnotetext{
${ }^{19}$ Zum Begriff der Prärogativen: TEZNER, Kaiser 1115; HuBER, Verfassungsgeschichte 11-18; speziell zu den militärischen Prärogativen des Kaisers: ScHMETTERER, Oberster Kriegsherr.

${ }^{20}$ Zur Abgrenzung von Kommandogewalt und Militärverwaltung: MARSCHALL VON BIEBERSTEIN, Verantwortlichkeit; zu dessen Auffassung: SCHMETTERER, Oberster Kriegsherr 13-14.

${ }^{21} \S 5$ DG, 1. Absatz.

${ }^{22}$ OlechOWSKI-HrdLICKA, Gemeinsame Angelegenheiten.

${ }^{23} \S 5$ DG, 2. Absatz.
}

Die Leitung, Führung und innere Organisation der Armee waren also Prärogativen des Kaisers. Diesbezüglich konnte er auch ohne Gegenzeichnung eines Ministers gültige Anordnungen treffen. ${ }^{24}$

Wie auch in anderen Bereichen entsprachen die Regelungen des ungarischen Ausgleichsgesetzes bezüglich der militärischen Befugnisse des Monarchen nicht exakt jener des österreichischen Delegationsgesetzes. $\S 11$ des ungarischen Gesetzesartikels 12:1867 lautete:

„Infolge der verfassungsmäßigen Herrscherrechte Seiner Majestät in Betreff des Kriegswesens wird all das, was auf die einheitliche Führung, Befehligung und innere Organisation der gesamten Armee [...] Bezug hat, als der Verfügung Seiner Majestät zustehend erkannt. “25

Gerade nach der Jahrhundertwende wurde diese Formulierung von vielen ungarischen Politikern (unter anderem im Neunerprogramm) so verstanden, dass ausnahmslos alle militärischen Anordnungen des Monarchen gegengezeichnet werden mussten. Das wurde mit dem Wort "verfassungsmäßig" begründet, aus dem auch hier eine Gegenzeichnungspflicht abgeleitet wurde. Dieser Standpunkt hätte bedeutet, dass es keine militärischen Prärogative des Monarchen gegeben hätte. Franz Joseph war aber nicht bereit, diesen Standpunkt zu akzeptieren. ${ }^{26}$

In der Praxis war es sogar so, dass der Kaiser ausnahmslos alle seine Anordnungen in Bezug auf die gemeinsame Armee ohne Gegenzeichnung des Kriegsministers traf. Die theoretische, konstitutionelle Unterscheidung zwischen Militärverwaltung und Kommandogewalt wurde in der Regierungspraxis bezüglich der gemeinsa-

\footnotetext{
${ }^{24}$ FRISCH, Verantwortlichkeit 356-365; SCHMID, Heeresrecht 11; ULBRICH, Lehrbuch 763.

${ }^{25}$ GA XII:1867, BERNATZIK, Verfassungsgesetze

Nr. 119.

${ }^{26}$ BERNATZIK, Verfassungsgesetze Nr. 178d; KOLMER, Parlament und Verfassung 507-508; SCHMID, Heerwesen 788-791.
} 
men Armee also nicht gemacht. ${ }^{27}$ Für beides war im Frieden der gemeinsame Kriegsminister zuständig und weder hier noch dort übernahm er durch Gegenzeichnung die Verantwortung für Anordnungen des Kaisers. Erst im Kriegsfall wurden beide Bereiche getrennt. Für die Kommandogewalt war das mit Kriegsbeginn errichtete Armeeoberkommando (AOK) zuständig, während die Zuständigkeit für die Militärverwaltung weiterhin beim Kriegsminister blieb. Trotz dieser organisatorischen Teilung blieb es aber dabei, dass beides monarchische Prärogativen waren, bei denen es keine ministerielle Gegenzeichnung gab.

Bei den beiden Landwehren war die Situation von Anfang an grundlegend anders. Hier waren Kommandogewalt und Militärverwaltung tatsächlich voneinander getrennt. In beiden Landwehren war ein unverantwortlicher Landwehroberkommandant im Frieden für Angelegenheiten der Kommandogewalt zuständig, während die Militärverwaltung von den verantwortlichen Landesverteidigungsministern erledigt wurde. Im Krieg übte dann das Armeeoberkommando die Kommandogewalt über die gesamte Bewaffnete Macht, also auch über die beiden Landwehren aus. ${ }^{28}$

\section{Kelsens Vorschläge}

Kelsen ging vor dem Hintergrund der ungarischen Forderungen davon aus, dass es nicht möglich sein würde, eine gemeinsame Armee beider Reichshälften auf Dauer zu erhalten. Anstelle des Kompromisses von 1868 mit der Dreiteilung in gemeinsame Armee und zwei Landwehren würde eine Teilung aller Truppen in eine österreichische und eine ungarische Armee

\footnotetext{
27 Pernthaler, Rechtsstaat und sein Heer 17.

${ }^{28}$ PAPP, Honved 645-646; SCHMETTERER, Oberster Kriegsherr 14-15; WAGNER, k.(u.)k. Armee, 368, 417422.
}

treten. Kelsen wollte nun trotz dieser organisatorischen Teilung eine möglichst große operative Einheit des österreichisch-ungarischen Militärs sichern. In seinem Aufsatz in der Zeitschrift für Militärrecht schlug er daher vor, die Militärverwaltung ganz auf die beiden Reichshälften aufzuteilen, die Kommandogewalt aber beim AOK als gemeinsamer Einrichtung zu belassen. Die beiden Landesverteidigungsminister sollten zu vollen Kriegsministern aufgewertet werden, das gemeinsame Kriegsministerium hingegen abgeschafft werden. ${ }^{29}$

Es ist nicht sonderlich überraschend, dass der gemeinsame Kriegsminister diesem Konzept nicht allzu viel abgewinnen konnte. Daher vollzog Kelsen eine gewisse Kehrtwende und schlug in seinen Gutachten für Stöger-Steiner nicht mehr vor, das gemeinsame Kriegsministerium abzuschaffen; stattdessen meinte er nun, dass es künftig im Frieden kein AOK mehr geben sollte. ${ }^{30}$ Insofern entsprachen Kelsens Vorschläge der Situation vor 1914. Insgesamt wollte Kelsen aber nicht zum Zustand vor dem Ersten Weltkrieg zurückkehren. Auch in seinen Gutachten für Stöger-Steiner schlug er vor, die Armee organisatorisch zwischen Österreich und Ungarn aufzuteilen und dafür die Landwehren abzuschaffen und die Landesverteidigungsministerien zu Kriegsministerien aufzuwerten. ${ }^{31} \mathrm{Au}-$ ßerdem sollte die Militärverwaltung schon gesetzlich klar von der Kommandogewalt unterschieden werden ${ }^{32}$ - und zwar so, dass in $\S 4$ des vorgesehenen Militärgesetzes alle Angelegenheiten der Kommandogewalt taxativ aufgezählt wurden und alles andere Militärverwaltung sein sollte.

Kelsen behielt sein Grundkonzept aus der Zeitschrift für Militärrecht in seinen Gutachten für Stöger-Steiner also weitgehend bei. Nur in ei-

\footnotetext{
${ }^{29}$ KELSEN, Reform.

${ }^{30}$ Gutachten 1, fol. $7^{\mathrm{r}-\mathrm{v}}$.

${ }^{31}$ Gutachten 1, fol. $9^{\text {r. }}$

${ }^{32}$ Gutachten 1, fol. $8^{\mathrm{r}-\mathrm{v}}$.
} 
nem Punkt revidierte er seine Vorschläge. Als zentrales gemeinsames Organ der geteilten Armeen sah er ursprünglich nur mehr das $\mathrm{AOK}$ vor, in seinen Gutachten dann aber nur mehr ein gemeinsames Kriegsministerium. Ein Nebeneinander von gemeinsamem Kriegsministerium und Armeeoberkommando hielt für nicht sinnvoll - jedenfalls nicht im Frieden. ${ }^{33}$

Es liegt nahe, dass Kelsen seine Meinung über die Beibehaltung eines gemeinsamen Kriegsministeriums nicht so sehr aus sachlichen, sondern eher aus institutionell persönlichen Gründen änderte. Trotzdem - oder gerade deshalb widmete er juristischen Argumenten für die Beibehaltung des gemeinsamen Kriegsministeriums in einem seiner Gutachten breiten Raum. ${ }^{34}$ Er meinte, dass ein gemeinsames Ministeriums zur Verwaltung der Kriegsmarine erforderlich wäre, weil die nicht zwischen den beiden Reichshälften aufgeteilt werden sollte. ${ }^{35}$ Weiters argumentierte Kelsen, dass das Budget für die gemeinsame Kommandogewalt von gemeinsamen Organen (den Delegationen) genehmigt wurde; daher sollte es seiner Meinung nach ein gemeinsames, verantwortliches Organ, also einen Kriegsminister geben, der einer parlamentarischen Kontrolle über die Verwendung dieses Budgets unterlag. ${ }^{36}$ Außerdem meinte Kelsen,

${ }^{33}$ Gutachten 1, fol. 9v.

${ }^{34}$ Gutachten 1, fol. $9^{\mathrm{r}-\mathrm{v}}$.

${ }^{35}$ Gutachten 1, fol. 9r; Ähnliches galt für die bosnischherzegowinischen Truppen. Kelsen erwähnte die bosnisch-herzegowinischen Truppen in seinem ursprünglichen Text jedoch nicht. Auf fol. $13^{v}$ findet sich lediglich folgender handschriftlicher Vermerk, vielleicht von Stöger-Steiner: „vom b[osnisch] $\mathrm{h}$ [erzegowinischen] Heereskontingent ist nirgends die Rede“. In der späteren Fassung des Gutachtens wurden dann auch die Verwaltung der bosnischherzegowinischen Truppen als Argument für ein gemeinsames Kriegsministerium genannt; OBERKOFLER, RABOFSKY, Kelsen im Kriegseinsatz 183.

${ }^{36}$ Gutachten 1, fol. $9^{\mathrm{r}-\mathrm{v}}$. Die Delegationen waren nur insofern gemeinsame Organe, als sie ausschließlich für die pragmatisch-gemeinsamen Angelegenheiten der Monarchie zuständig waren; sie tagten aber in der dass eine gemeinsame Verwaltungsstelle erforderlich wäre, um die Einheitlichkeit der Militärverwaltung sicherzustellen. ${ }^{37}$ Schließlich verwies er darauf, dass es in der Vergangenheit zwar auch im Kriegsfall Armeeoberkommanden gegeben hatte, diese im Frieden aber regelmäßig bald wieder aufgelöst worden waren. ${ }^{38}$

Insgesamt sind Kelsens Argumente nicht sehr überzeugend. Eine Aufteilung der Kriegsmarine auf die beiden Reichshälften war nicht prinzipiell unmöglich. ${ }^{39}$ Besonders schwach wirkt Kelsens Verweis auf die notwendige budgetäre Verantwortlichkeit des gemeinsamen Kriegsministers. Bei einer Teilung der Armee hätte man die Budgethoheit wohl vollständig an die Parlamente der beiden Reichshälften übertragen können; und selbst wenn das nicht geschehen wäre, wäre die Verantwortlichkeit des gemeinsamen Kriegsministers ohne die Erlassung von Ausführungsgesetzen weiter eine rein theoretische Sache geblieben. Auch von der notwendigen Einheitlichkeit der Militärverwaltung wird Kelsen nicht sonderlich überzeugt gewesen sein - sonst hätte er nicht kurz zuvor noch geschrieben, dass eine völlige Aufteilung der Militärverwaltung auf beide Reichshälften möglich und sinnvoll gewesen wäre.

Für Kelsen war die Aufteilung der Militärverwaltung noch aus einem anderen Grund attraktiv, der mit den traditionellen national-un-

Regel nicht gemeinsam. Auf die Besonderheiten und Schwierigkeiten, die sich daraus ergaben, ging Kelsen in seine Gutachten zur Armeereform nicht weiter ein. $\mathrm{Zu}$ den Delegationen: HöBELT, Delegations; OlechOWSKI-HRDLICKA, Gemeinsame Angelegenheiten 361-426; SOMOGYI, Delegation.

37 Gutachten 1, fol. 9v.

${ }^{38}$ Gutachten 1, fol. $10^{\mathrm{r}-\mathrm{v}}$.

${ }^{39}$ Bei den bosnisch-herzegowinischen Truppen wäre eine Eingliederung in die österreichische oder ungarische Armee ohne Änderung des staatsrechtlichen Status' Bosniens allerdings schwieriger gewesen; aber genau dieses Argument war in Kelsens ursprünglicher Fassung des Gutachtens noch gar nicht enthalten. 
garischen Forderungen nach einer Teilung der Armee nur wenig zu tun hatte. Ihm war nämlich eine Verrechtlichung des Militärs, eine starke Eingliederung der Armee in die allgemeinen staatlichen Strukturen ein großes Anliegen..$^{40}$ Seine Opposition zur Armee als „Staat im Staate", seine Forderung, dass auch Soldaten Staatsbürger sein sollten ist gerade angesichts der späteren Entwicklungen in Deutschland bemerkenswert. Immerhin wurde an der Reichswehr der Weimarer Republik deren Selbstverständnis als "Staat im Staate" stark kritisiert, ${ }^{41}$ und gerade deshalb in der Bundeswehr nach dem Zweiten Weltkrieg das Konzept vom „Staatsbürger in Uniform" betont. ${ }^{42}$

Ein zentrales Instrument zur Verrechtlichung der Militärverwaltung war für Kelsen die Einbeziehung des militärischen Bereichs in die Verwaltungsgerichtsbarkeit. ${ }^{43}$ Bisher hatte es im Habsburgerreich in militärischen Angelegenheiten keinen verwaltungsgerichtlichen Rechtsschutz gegeben. In Cisleithanien gab es zwar seit 1876 einen Verwaltungsgerichtshof, die pragmatischen und dualistischen Angelegenheiten und damit der Großteil der militärischen Angelegenheiten - waren aber ausdrücklich aus dessen Wirkungsbereich ausgenommen. ${ }^{44}$ Einen gemeinsamen Verwaltungsgerichtshof gab es aber nicht, und nach Kelsens - wohl zutreffender Einschätzung - wäre die Einrichtung eines solchen politisch nicht durchsetzbar gewesen. Es ist schwer vorstellbar, dass Ungarn einer zusätzlichen gemeinsamen Behörde zugestimmt hätte. Hätte man hingegen die gesamte Militärverwaltung zwischen den beiden Reichshälften aufgeteilt, wäre es leicht gewesen, die Zuständigkeit des cisleithanischen Verwaltungsgerichtshofs auf die gesamte cisleithanische Militärverwal-

\footnotetext{
${ }^{40}$ Gutachten 2, pag. 6.

${ }^{41}$ Zur Reichswehr: WOHLFEIL, DOLLINGER, Reichswehr.

${ }^{42}$ Zur Bundeswehr siehe nur: BALD, Bundeswehr.

${ }^{43}$ Gutachten 1, fol. 13v .

${ }^{44}$ RGBl. 36/1876; § 3 lit. c-d.
}

tung auszudehnen und so Kelsens Vorstellung von einer Verrechtlichung des Militärwesens zu verwirklichen.

Kelsens Entwurf für ein Militärgesetz dürfte nicht in jeder Hinsicht seinen Idealvorstellungen einer neuen Wehrverfassung für ÖsterreichUngarn entsprochen haben. Ein bisschen wirkt er wie der Versuch, die Quadratur des Kreises zu lösen: Erstens wollte er die Armee organisatorisch teilen und gleichzeitig ihre operative Einheit sicherstellen, zweitens versuchte eine weitgehende Teilung der Militärverwaltung unter Beibehaltung des gemeinsamen Kriegsministeriums. Trotzdem gelang es Kelsen, ein in sich konsistentes Gesetz vorzuschlagen, das technisch sauber gearbeitet war. Wäre sein Vorschlag so umgesetzt worden, hätte er zumindest die Unklarheiten und Widersprüche der Gesetze von 1867 vermieden. Ob sich Kelsen Konzept sonst bewährt hätte, lässt sich nicht sagen - es kam nie zur praktischen Umsetzung.

Kelsen stellte nicht nur Überlegungen an, wie die österreichisch-ungarische Wehrverfassung inhaltlich reformiert werden sollte, sondern auch, wie militärischen Interessen bei Reformen gewahrt werden konnten. Dazu schlug er vor, eine besondere militärisch-juristische Stelle einzurichten. Er spezifizierte dabei nicht, wie diese Stelle heißen oder wo genau sie eingerichtet werden sollte, skizzierte aber, mit welchen Fragen sie sich beschäftigen sollte, und gab damit einen kurzen Überblick über die aus seiner Sicht besonders wichtigen Reformfragen..$^{45}$ Der Verdacht liegt nahe, dass Kelsen die Errichtung einer neuen militärrechtlichen Fachstelle auch deshalb vorschlug, weil er hoffte, dass sich in diesem Zusammenhang eine interessante Position für ihn selbst ergeben würde.

Die von ihm vorgeschlagene neue Stelle wurde nicht errichtet, aber mit seinem nächsten Vorschlag hatte Kelsen mehr Erfolg. In einer Denk-

${ }^{45}$ Gutachten 2. 
schrift vom 6. März 1918 schlug er dem Kriegsminister und anderen Stellen die Errichtung einer eigenen Professur für Militär-Verfassungsund Verwaltungsrecht vor, wobei er bei der Besetzung dieser neuen Professur offenbar an sich selbst dachte. ${ }^{46}$ Tatsächlich wurde Kelsen durch kaiserliche Entschließung vom 6. Juli mit Wirkung zum 1.Oktober 1918 zum außerordentlichen Professor für Staats- und Verwaltungsrecht unter besonderer Berücksichtigung des Militärrechts ernannt. ${ }^{47}$ Das Militärrecht hatte also eine wichtige Rolle auf Kelsens Weg zur Professur gespielt. Trotzdem beschäftigte er sich nach dem Ende des Ersten Weltkriegs praktisch nicht mehr mit militärrechtlichen Fragen.

\section{Gutachten 1}

28-seitiges Typoskript mit Randschriften (in rot), Streichungen, hand- und maschinschriftlichen Ergänzungen, ÖNB, Nachlass Stöger-Steiner, Cod. Ser. n. 54574;

Unterstreichungen im Original wurden übernommen; später gestrichene Passagen wurden durch Kursivsetzung gekennzeichnet; die Randschriften wurden in eckige Klammern gesetzt. Die maschin- und handschriftlichen Ergänzungen wurden als Fußnoten wiedergegeben; die handschriftlichen Ergänzungen stammen nicht von Kelsen, sondern vermutlich von StögerSteiner. Offenkundige Schreibfehler wurden korrigiert.

Das vorliegende Typoskript ist eine frühere Fassung von Dokument III in OBERKOFLER, RABOFSKY, Kelsen im Kriegseinsatz; in der dort edierten Version sind die hier vorgenommen Streichungen und handschriftlichen Ergänzun-

\footnotetext{
${ }^{46}$ OBERKOFLER, RABOFSKY, Kelsen im Kriegseinsatz 195-197 (Dokument V).

47 OberKofler, RABOFSKy, Kelsen im Kriegseinsatz 9597
}

gen - wenn auch nicht durchwegs im genauen Wortlaut-berücksichtigt.

Dem Typoskript in der ÖNB ist der erwähnte Militärgesetz-Entwurf nicht angeschlossen. Da Kelsens Gutachten direkt Bezug auf diesen Entwurf nimmt, ist er im Folgenden nach der Edition bei Oberkofler, Rabofsky ${ }^{48}$ wiedergegeben:

\section{Text}

Vertraulich!!!

K.k. österr. Ministerpräsident (Person) ....1

K.u. ungar. Ministerpräsident (Person) ......2 Wien, am 9. Jänner 1918.

1 und 2.

\section{EUER EXZELLENZ}

beehre ich mich im Folgenden die Grundzüge einer geplanten Reform der Wehrmachtsorganisation bekanntzugeben, auf Grund welcher ich im Sinne des Allerhöchsten Befehlsschreiben vom 8. dieses Monats mit den Ministerpräsidenten beider Staaten der Monarchie in Verhandlungen zu treten habe. Denn die geplante Reform geht über den Bereich des SEINER MAJESTÄT nach den Verfassungen beider Staaten der Monarchie gewährleisteten Reservatrechtes der inneren Organisation der Wehrmacht hinaus; sie berührt die gesetzlichen Grundlagen der Wehrmacht, kann daher nur im vollen Einvernehmen mit den Regierungen beider Staaten in Angriff genommen und nur unter der Mitwirkung der Legislativen beider Staaten durchgeführt werden.

I.

[Die Reformbedürftigkeit der verfassungsrechtlichen Grundlagen.] Es ist eine bekannte Tatsache, daß die beiden Ausgleichsgesetze vom Jahre 1867, auf denen die Wehrmachtsorganisation staatsrechtlich beruht, und die dem Sinne des Ausgleichswerkes nach der Ausdruck zweier im Wesen übereinstimmender Staatswillen über ihr

${ }^{48}$ Oberkofler, RABOfSKy, Kelsen im Kriegseinsatz 187-190 
gegenseitiges Verhältnis sein sollten, in nicht unerheblichem Maße mit einander in Widerspruch stehen und daß gerade in der Regelung des Kriegswesens hinsichtlich Art und Umfang der Gemeinsamkeit [2 ${ }^{\mathrm{r}}, 1$. Einlagebogen] die bedeutendsten Differenzen bestehen.

Um nur die wesentlichsten, in der Praxis der Kriegsverwaltung seit jeher besonders hart empfundenen Mängel hervorzuheben, sei daran erinnert, daß für das Kriegswesen, als einer sogenannten pragmatischen Angelegenheit im österreichischen Ausgleichsgesetze eine Gemeinsamkeit sowohl hinsichtlich der Verwaltung als auch der Gesetzgebung prinzipiell statuiert ist. [Die Gesetzgebung auf dem Gebiete des Kriegswesens.] Was speziell die Gesetzgebungsgemeinsamkeit betrifft, die gerade für das Kriegswesen von größter Bedeutung ist, weil auf diesem Gebiete mehr als auf denen der übrigen pragmatischen Angelegenheiten Gesetze als Grundlage der Verwaltung in Betracht kommen, so ist diese in der Weise geregelt, daß das bezügliche Gesetzgebungsrecht den Delegationen übertragen ist ( $\$ 6$ des Gesetzes vom 21./12. 1867, RGBl. 146), wodurch eine Garantie dafür geschaffen werden sollte, daß solche Gesetze nur in inhaltlicher Übereinstimmung für beide Staaten der Monarchie zustande kommen können. (§ 15 des zitierten Gesetzes). Das ungarische Ausgleichsgesetz räumt jedoch - wie nach seinem in diesem Punkte allerdings nicht ganz eindeutigen Texte angenommen wird, - den Delegationen ein materielles Gesetzgebungsrecht nicht ein. Es werden daher Gesetze auf dem Gebiete des Kriegswesens nicht von den Delegationen, und daher nicht unter dieser von dem österreichischen Ausgleichsgesetze geschaffenen Garantie der inhaltlichen Übereinstimmung in beiden Staaten von den beiden Parlamenten beschlossen. ${ }^{49}$ Die - offenbar nicht

\footnotetext{
${ }^{49}$ Die Heeresverwaltung kann sich weder die nur auf das ungar. Ausgleichsgesetz, noch die nur auf das österr. Ausgleichsgesetz gestützten Argumentationen
}

vorhergesehene und nicht beabsichtige - Folge davon ist, daß lediglich die zu sogenannten dualistischen Angelegenheiten zählende Feststellung des Wehrsystems (§2, Pkt. 5 des österreichischen Ausgleichsgesetzes) kraft der ausdrücklich [2v] hiefür in beiden Ausgleichsgesetzen vorgesehenen Behandlung inhaltlich übereinstimmend erfolgen muß, daß aber für jedes andere militärische Gesetz eine verfassungsrechtliche Garantie für die Bindung seines Inhalts an den eines korrespondierenden Gesetzes im andern Staate kaum nachweisbar ist. Jedenfalls können sich alle bezüglichen Versuche nur auf sehr schwache Argumente stützen. ${ }^{50}$ Der pragmatischen Verwaltungsgemeinsamkeit steht somit keineswegs die erforderliche inhaltliche Übereinstimmung der beiderstaatlichen Gesetze gegenüber, auf Grund deren die Verwaltung zu erfolgen hat. Dazu kommt noch, daß auch hinsichtlich der Feststellung des Wehrsystems speziell von ungarischer Seite die Notwendigkeit einer prinzipiellen Übereinstimmung der bezüglichen Wehrgesetze in Frage gestellt wird.

[Der Umfang der Verwaltungsgemeinsamkeit.] Nach dem klaren und unzweideutigen Wortlaut des österreichischen Ausgleichsgesetzes ist prinzipiell das ganze Kriegswesen als gemeinsam anerkannt. Ausgenommen von dieser Gesetzgebungs- und Verwaltungsgemeinsamkeit sind lediglich bestimmte, im $\S 1$, Punkt b ausdrücklich angeführte Angelegenheiten: Rekrutenbewilligung, Gesetzgebung über die Art und Weise der Erfüllung der Wehrpflicht, Verfügungen hinsichtlich der Dislozierung und Verpflegung des Heeres, Regelung der bürgerlichen Verhältnisse

zu eigen machen. - Da mangels einer einheitlichen Voraussetzung mit juristischen Argumentationen überhaupt kein fruchtbares Resultat zu erzielen ist, muß sich die Heeresverwaltung auf den politischen Gesichtspunkt loyaler, die Interessen beider Staaten und das Gesamtinteresse der Monarchie gleichmäßig berücksichtigender Parität beschränken, weil nur dadurch das militärische Interesse, nämlich die Aufrechterhaltung und Stärkung der Verteidigungsfähigkeit der Monarchie gewahrt bleibt. [handschriftlich]. ${ }^{50}$ Hier sei besonders an den Streit wegen der Behandlung der Militärjustizgesetze erinnert. [maschinschriftlich]. 
und der sich nicht auf den Militärdienst beziehenden Rechte und Verpflichtungen der Mitglieder des Heeres. Es handelt sich dabei also offenbar nur um Ausnahmen von der Gesetzgebungsgemeinsamkeit. Lediglich bei den "Verfügungen" hinsichtlich Dislokation und Verpflegung könnte zweifelhaft sein, ob nicht auch eine Ausnahme von der Verwaltungsgemeinsamkeit vorliegt. Von dieser - übrigens durchaus zweifelhaften - [3r] Ausnahme abgesehen stellt der Wortlaut des österreichischen Ausgleichsgesetzes prinzipiell das ganze Kriegswesen in Verwaltungsgemeinsamkeit, scheint also eine andere als eine gemeinsame Militärverwaltung nicht zu kennen. Innerhalb des in Verwaltungsgemeinsamkeit stehenden Kriegswesens nimmt eine besondere Stellung nach $\S 5$, Abs. 2 des österreichischen Ausgleichsgesetzes nur jener Teil ein, der als Leitung, Führung und innere Organisation der gesamten Armee bezeichnet ist. Während nämlich die übrige Verwaltung durch einen gemeinsamen verantwortlichen Minister als Mitglied des gemeinsamen verantwortlichen Ministeriums (§5, Abs. 1) zu erfolgen hat, steht die Anordnung hinsichtlich der Leitung, Führung und inneren Organisation der gesamten Armee ausschließlich dem Kaiser zu, das heißt, die auf solche Weise umschriebene Allerhöchste Kommandogewalt wird nicht durch einen verantwortlichen Minister und sohin unter parlamentarischer Kontrolle, sondern unverantwortlich, weil unmittelbar durch den Kaiser ausgeübt.

Nach dem Wortlaute des ungarischen Ausgleichsgesetzes, das in diesem Punkte die auffallendste Differenz gegenüber dem österreichischen aufweist, könnte ernstlich in Zweifel gezogen werden, ob ebenso wie im österreichischen Delegationsgesetze die prinzipielle Gemeinsamkeit des ganzen Kriegswesens ausgesprochen ist. §9 des Gesetzartikels XII erklärt das "Kriegswesen" schlechtweg, das heißt doch wohl das ganze Kriegswesen, nicht etwa als eine gemeinsame Angelegenheit, sondern als ein "Mittel der gemeinsamen Verteidigung." Damit ist keineswegs klar genug gesagt, daß das ganze Kriegswesen - so wie im österreichischen Ausgleichsgesetz - prinzipiell als eine gemeinsame Angelegenheit im Sinne einer das ganze Kriegswesen umfassenden Verwaltungsgemeinsamkeit anerkannt sei. Als ein Mittel der gemeinsamen und simultanen Verteidigung (\$2 des Gesetzartikels XII spricht von ei[3v]ner gemeinsamen und "wechselseitigen" Verpflichtung zur Verteidigung) wird im § 8 auch die zweckmäßige Leitung der auswärtigen Angelegenheiten und zwar aller auswärtigen Angelegenheiten erklärt. Eine Gemeinsamkeit der Verwaltung wird jedoch - auch hier zum Unterschied vom österreichischen Ausgleichsgesetznur für bestimmte, nicht für alle auswärtigen Angelegenheiten statuiert. Die gemeinsame oder "wechselseitige" Verteidigungspflicht ist keineswegs mit der Gemeinsamkeit des Kriegswesens identisch. Zweifellos fällt die erst nach dem Ausgleichsgesetz geschaffene Honvéd, ebenso wie die österreichische Landwehr in die gemeinsame oder wechselseitige Verteidigungspflicht, obgleich die k.u. Honvéd und die k.k. Landwehr keine gemeinsamen, das heißt gemeinsam verwalteten Institutionen sind. Der Wortlaut der Bestimmungen der §§ 10-14 des Gesetzartikels XII kann sicherlich den Eindruck erwecken, daß die Gemeinsamkeit des Kriegswesens sich nur auf diejenigen Angelegenheiten erstreckt, die in diesem $\mathrm{Zu}$ sammenhange ausdrücklich angeführt sind, somit also auf die im $\S 11$ in gleicher Weise wie im österreichischen Ausgleichsgesetz umschriebene Allerhöchste Kommandogewalt. Zweifellos lag dies nicht in den Absichten der Schöpfer des Gesetzartikels XII. Diese wollten offenbar einen faktisch bestehenden Zustand aufrechterhalten: die einheitliche Armee einschließlich Kommandogewalt und Militärverwaltung. Allein man übersah offenbar, daß der bestehende Rechtszustand nicht aufrechterhalten werden konnte, sondern daß vielmehr eine völlig neue Rechtsgrundlage zu schaffen gewesen wäre. So wie durch den Ausgleich von 1867 die Monarchie aus einem Einheitsstaat zu zwei souveränen und gleichberechtigten Staaten, so mußte die Armee aus dem Organ eines Einheitsstaates zu der gemeinsamen Armee zweier Staaten umgestaltet [4r, 2. Einlagebogen] werden. Dieses gesetzestechnische Problem hat der Gesetzartikel XII ungelöst gelassen und durch sein Stillschweigen zu den widersprechendsten Interpretatio- 
nen Anlaß gegeben. Dadurch, daß der Gesetzartikel XII überdies die Bezeichnung "gemeinsames" Heer geflissentlich vermeidet, und sogar ausdrücklich von einem "ungarischen Kriegsheer" spricht, ist für die staatsrechtliche Gemeinsamkeit des Heeres und seiner Verwaltung im ungarischen Ausgleichsgesetz sofern man nur seinen Wortlaut ins Auge faßt - so gut wie keine Stütze zu finden. ${ }^{51}$ Und dem gegenüber ist der immer wieder versuchte Rekurs auf die erweisliche Absicht der Gesetzgeber, die bei den §§ 10-14 des Gesetzartikels XII ein gemeinsames Heer in gemeinsamer Verwaltung vor Augen hatten, ist die Berufung auf die historisch gewordenen Aussprüche Deák's und Andrassys insbesondere hinsichtlich der Bedeutung des Ausdruckes "ungarisches Kriegsheer" von verhältnismäßig geringer Bedeutung. Denn der zu diesen Absichten der Gesetzgeber in unbegreiflichem Widerspruch stehende Wortlaut des Gesetzes wird immer wieder von mächtigen Parteien als Argument für ihre auf Negierung der faktischen Gemeinsamkeit gerichteten Bestrebungen benützt und gerade diese Parteien stehen heute der Regierung nahe. Auch kann sich die Heeresverwaltung nicht auf die Kraft juristischer Argumentationen stützten, zumal wenn sie auf einem so unsicheren Grunde ruhen, wie es die beiden Ausgleichsgesetze sind, sondern nur auf reale politische Tatsachen. Sie muß mit den Kräften rechnen, die hinter den - von anderer Seite vielleicht für unrichtig erklärten - juristischen Argumentationen stehen:

[Die staatsrechtliche Stellung der Kommandogewalt] Nicht nur hinsichtlich des Umfangs der Gemeinsamkeit, auch über die Art derselben insbesondere mit

\footnotetext{
${ }^{51}$ Sie kann sich weder die nur auf das ungarische Ausgleichsgesetz noch die auf das österreichische Ausgleichsgesetz gestützte Argumentationen zu eigen machen. Da mangels einer einheitlichen Voraussetzung mit juristischen Argumentationen überhaupt kein fruchtbares Resultat $z u$ erzielen ist, muß sich die Heeresverwaltung auf den politischen Gesichtspunkt loyaler, die Interessen beider Staaten und das Gesamtinteresse der Monarchie gleichmäßig berücksichtigender Parität beschränken. [maschinschriftlich, entspricht im Wesentlichen der handschriftlichen Ergänzung auf fol. $2^{\mathrm{r}}$.
}

Beziehung auf die staatsrechtliche [4v ${ }^{\mathrm{v}}$ Stellung der Kommandogewalt läßt der Gesetzartikel XII höchst bedenkliche Zweifelsmöglichkeiten offen. Auch in diesem Punkte bleibt sein Wortlaut hinter der anderweitig geäußerten Ansicht seiner Gesetzgeber zurück. Aus der Entstehungsgeschichte des $\$ 11$ geht mit Sicherheit hervor, daß man die Verfügung über das gestellte Heer ausschließlich und unmittelbar dem König übertragen und nur auf die sachliche und persönliche Bereitstellung des Heeres dem Parlamente den konstitutionellen Einfluß einräumen wollte. Nichtsdestoweniger ist es wieder der Wortlaut des $\S 11$, die in seinen Einleitungsworten erfolgenden Beziehung auf die "verfassungsmäßigen Herrscherrechte", auf die gestützt eine Ansicht vertreten wird, der zufolge im direkten Widerspruch zu der bezüglichen Bestimmung des österreichischen Ausgleichsgesetzes jedem wirklichen Reservatrecht der Krone auf dem Gebiete des Kriegswesens der Boden entzogen wird. ${ }^{52}$ Dabei befindet sich die Kriegsverwaltung in der seltsamen Situation, daß, wenn sie die im $\S 11$ erwähnte Führung, Befehligung und innere Organisation den Intentionen der Gesetzgeber entsprechend als die SEINER MAJESTÄT unter Ausschluß ministerieller Verantwortung und parlamentarischer Kontrolle reservierte Kommandogewalt festhält, ernstlich Gefahr läuft, die letzte Möglichkeit zu verlieren, die gemeinsame Militärverwaltung, die Kompetenz eines gemeinsamen verantwortlichen Kriegsministers, (den der Gesetzartikel XII in auffallendem Gegensatz zu der ausdrücklichen Nennung des gemeinsamen Ministers des Äußern oder gemeinsamen Finanzministers nicht anführt), in dem Wortlaut des ungarischen Ausgleichsgesetzes zu begründen. Denn dies scheint nur dann möglich zu sein, wenn unter Führung, Befehligung und innere Organisation neben der Allerhöchsten Kommandogewalt auch [5 $\left.5^{\mathrm{r}}\right]$ die Militärverwaltung verstanden wird. 53

\footnotetext{
${ }^{52}$ Das bekannte 9-er Programm vom Jahre 1903 spricht in seinem Punkt 8 diesen Gedanken geradezu aus. [maschinschriftlich].

${ }^{53}$ Ein Widerspruch, aus dem es kaum einen logischen Ausweg gibt! [maschinschriftlich].
} 
[Die politischen Kämpfe um die Militärverfassung.] Angesichts dieses wohl beispiellosen Zustandes der verfassungsrechtlichen Grundlagen der Wehrmacht ist es nicht zu verwundern, daß um die prinzipiellsten Voraussetzungen ihres Bestandes als gemeinsamer Institution vom ersten Tage nach Perfektuierung des Ausgleiches bis mitten in den Weltkrieg hinein die schwersten Verfassungskämpfe geführt wurden. In welchem Maße diese die Grundfesten der Monarchie erschütternden Kämpfe das Verhältnis zwischen beiden Staaten getrübt und jede ruhige Entwicklung des Kriegswesens ${ }^{54}$ unterbunden ${ }^{55}$ haben, weiß jeder, dem die Leidensgeschichte unserer Wehrmacht, zugleich das Hauptkapitel in der inneren Verfassungsgeschichte Österreich-Ungarns bekannt ist. Erinnert sei nur an die furchtbaren Krisen des Jahres 1903, und 1905/6, an die schweren Schädigungen, die der Wehrmacht durch die zeitweise Verweigerung des Rekrutenkontigentes zugefügt wurden.

[Die Verfassungswidrigkeit der tatsächlichen Gestaltung.] Die gänzliche Unzulänglichkeit der staatsrechtlichen Basis, auf der die Organisation des Kriegswesens beruht, macht es auch begreiflich, daß die tatsächliche Gestaltung der Wehrmacht, zwei einander widersprechenden Gesetzen gegenüber, sich niemals in einen Zustand voller Verfassungsmäßigkeit befinden kann. Je mehr sie sich dem einen Gesetze nähert, desto mehr muß sie sich vom anderen entfernen. Die Unsicherheit der Grundlagen, die für die Gemeinsamkeit des Kriegswesens gelegt wurden, [Der Abbau der Gemeinsamkeit.] hat zur Folge, daß unter dem Drucke politischer Verhältnisse diese Gemeinsamkeit schon vom ersten Tage ihres faktischen Bestandes an stückweise abzubröckeln begann. Kaum ein Jahr nach der Vollendung des Ausgleichswerkes wurden die beiden Landwehren geschaffen. Ursprüng-

\footnotetext{
${ }^{54}$ u. damit die militär. Kraft der Monarchie [handschriftlich].

${ }^{55} \mathrm{u}$ die Großmachtstellung der Monarchie in Frage gestellt [handschriftlich].
}

lich als eine Art Reserve gedacht, [5] haben sie sich bald $\mathrm{zu}$ vollwertigen stehenden Heeren entwickelt, so daß die Monarchie an Stelle einer Armee nunmehr deren drei besitzt. Eine Entwicklung, die weder mit dem Geiste des Ausgleiches, noch mit dem Wortlaute beider Ausgleichsgesetze, deren Text während dieser Zeit unverändert blieb, in Einklang gebracht werden kann; eine Entwicklung, die aber auch eine ganz außerordentliche Komplikation der auf drei große Zentralstellen verteilten Militärverwaltung mit sich brachte.

Der den Gedanken der Gemeinsamkeit stetig aushöhlende Prozeß ergriff aber auch den noch gemeinsam verbliebenden Teil des Kriegswesens. In diesem Zusammenhange seien nur die zum größten Teile erfüllten Forderungen des sogenannten 9-er Programmes erwähnt, und die Tatsache festgestellt, daß mit der Kommandierung von Offizieren ungarischer Staatsbürgerschaft zu den ungarischen Truppen des gemeinsamen Heeres innerhalb dieses Körpers schon jetzt ein spezifisch ungarischer und ein spezifisch östereichischer Bestandteil geschaffen wurde und daß in der zuletzt erfolgten Regelung der Fahnenfrage der Ausdruck der Gemeinsamkeit auf ein Minimum reduziert wurde. Wer seine Augen vor Tatsachen nicht verschließt, muß erkennen, daß der Abbau der Gemeinsamkeit auf dem Gebiete der Militärverwaltung einen Punkt erreicht hat, von dem aus es kein Zurück mehr gibt, auf dem zu beharren aber mangels jeder gesellschaftlichen Stütze kaum möglich und wegen der Halbheit und Zweckwidrigkeit des Zustandes auch nicht wünschenswert ist. Schon seit Jahren und längst vor diesem Krieg war jedem, dem die Wahrung militärischer Interessen oblag, die dringende Notwendigkeit einer Reform der verfassungsrechtlichen Grundlagen der Wehrmacht offenkundig. [6r, 3. Einlagebogen] Die Heeresverwaltung musste aus dieser unerträglichen Situation allgemeiner Unsicherheit heraus, mußte diesem beschämenden Abgleiten auf schiefe Ebene ein Ende machen und zu festen und klaren Verhältnissen gelangen. 
[Das Aufleben der Armeefrage im Kriege.] Jedoch noch bevor eine solche Konsolidierung der Verhältnisse in Angriff genommen werden konnte, brach der Krieg aus. So wie er ${ }^{56}$ in Österreich das Staatsgefühl gestärkt und der staatlichen Organisation, dadurch daß sie sich gegen eine Welt von Feinden militärisch $\mathrm{zu}$ behaupten vermochte, das Selbstbewusstsein gehoben hat, ${ }^{57}$ so hat er auch in Ungarn den Nationalstolz hoch aufschäumen lassen. Es ist nur natürlich und vom Standpunkte militärischer Interessen zu begrüßen, daß im Kriege das gesteigerte National- und Staatsgefühl vor allem in der Armee seinen Ausdruck sucht. Die Armee, die im Kriege wie in einem Brennpunkte alle Kräfte des Volkes vereinigt, ist für seine unmittelbare Anschauung die sinnfällige Verkörperung seines Willens zu ehrenvoller Selbstbehauptung. Man kann diese geistige Bewegung, die das Schwergewicht des nationalen Bewußtseins in die Armee verlegt, bei allen kriegführenden Staaten, ja auch bei solchen konstatieren, die früher der militärischen Organisation nur eine geringe Bedeutung im öffentlichen Leben eingeräumt hatten, wie England und Amerika. Es ist daher durchaus verständlich, daß in Ungarn der alte Wunsch nach einer nationalen Form des Heeres ${ }^{58}$ gerade in diesem lang währenden Kriege wieder auflebt, daß die Forderung erhoben wird, die pragmatische Gemeinsamkeit des Kriegswesens, die wechselseitige Verteidigungspflicht beider Staaten möge sich mit einer Organisation vereinbar erweisen, in der die Souveränität das ungarischen - ebenso wie des österreichischen [6 ${ }^{\mathrm{v}}$ ] Staates -, in der der besondere Charakter beider Staaten auch äußerlich zum Ausdrucke kommen.

[Die Notwendigkeit staatsrechtlich befriedigender Formen der Wehrmachtsorganisation.] Vom Standpunkte militärischer Interessen und insbesondere vom Standpunkte der gemeinsamen Kriegs-

\footnotetext{
${ }^{56}$ Als nun der Krieg ausbrach hat er [handschriftlich].

${ }^{57}$ und [handschriftlich].

${ }^{58}$ nach dem im Ausgleichsgesetz erwähnten ung. Heer [handschriftlich].
}

verwaltung, ist es in höchstem Grade wünschenswert, 59 muß es gerade als eine Lebensnotwendigkeit der Armee bezeichnet werden, daß ${ }^{60}$ die Organisation der Wehrmacht sich in Grundformen bewege, die ebenso den Bedürfnissen der Monarchie nach Einheit und hemmungslosem Zusammenwirken aller militärischen Kräfte, wie insbesondere auch den berechtigten, durch ihr notwendiges gegenseitiges Verhältnis abgestimmten Sonderinteressen beider Staaten entsprechen. Wenn die bisherigen Formen der Gemeinsamkeit diese Sonderwünsche beider Staaten - oder auch nur eines von ihnen nicht mehr befriedigen, muß eben eine andere Form gesucht werden. Es hat wenig Zweck an traditionellen Formen allzu starr festzuhalten, hinter denen sich der Inhalt schon allmählich so geändert hat, daß er die Form zu sprengen droht; zumal wenn durch solch intransigentes Festhalten der Schein entstehen könnte, daß die hergebrachten Formen der Gemeinsamkeit sehr im Interesse des festhaltenden als des die Änderung fordernden Teiles stehen, was ja dem Geiste des Ausgleiches nicht entspräche.

Eine die nationalen Interessen befriedigende Form der Wehrmachtsorganisation ist vom Standpunkte der Kriegsverwaltung schon darum zu wünschen, weil sie es ermöglicht, ${ }^{61}$ die Volkskräfte ungehemmt in die Bahnen militärischer Verteidigung strömen lassen, weil durch sie der peinlichste Gegensatz in dem Verhältnis beider Staaten beseitigt und dadurch die Kraft der Monarchie sohin auch ihre Wehrmacht gestärkt ${ }^{62}$ wird. Dies alles unter der Voraussetzung: [7 $\left.{ }^{\mathrm{r}}\right]$ daß es gelingt, bei gleichzeitiger Befriedigung der nationalen Aspirationen die Organisation der Wehrmacht auf eine gesunde, feste Basis zu

\footnotetext{
59 ja [handschriftlich].

${ }^{60}$ die nationale Begeisterung für die Zwecke der milit. Verteidigung der Monarchie erhalten, ihnen dienstbar gemacht wird. Dies - wird aber nur stattfinden, wenn [handschriftlich].

${ }^{61}$ wird [handschriftlich].

${ }^{62}$ en [handschriftlich].
} 
stellen und noch bessere Garantien für die Einheitlichkeit und ${ }^{63}$ Schlagkraft der Wehrmacht zu schaffen, als bisher bestanden haben.

[Die Erfahrungen des Krieges.]Für die Lösung dieses Problems, vor das die Heeresverwaltung zu ihrem Bedauern mitten im Kriege gestellt wurde, hat gerade dieser Krieg wertvolle Erfahrungen beigebracht. Er hat gezeigt, daß administrativ getrennte Armeekörper, die nach ihrer Staatsangehörigkeit und Sprache gänzlich verschieden waren, dennoch unter einheitlichem Kommando durchaus befriedigend $\mathrm{zu}$ einheitlichen strategisch-taktischen Zielen zusammenwirken können. Es sind insbesondere die Erfahrungen, die das k.u.k. Armeeoberkommando mit den beiden Landwehren und speziell mit den in ungarischer Sprache kommandierten und administrierten Honvéds hinsichtlich ihrer klaglosen Einfügung in das Ganze der österreichisch-ungarischen Wehrmacht gemacht hat, die denkbar besten.

All diese Erfahrungen lassen die militärpolitischen Wünsche der ungarischen Nation kühler und nüchterner beurteilen als dies noch vor dem Kriege der Fall war, lassen die beruhigende Überzeugung reifen, daß diese Wünsche unter voller Wahrung des Gesamtinteresses der Monarchie und sohin auch des Interesses des anderen Staates erfüllt werden können.

[Die entscheidende Formel.] Die für die Lösung des Problems entscheidende Form ist gefunden und die gestellte Aufgabe befriedigend gelöst, wenn die nach den Verfassungen beider Staaten ausschließlich SEINER MAJESTÄT zustehende der ministeriellen Verantwortlichkeit und parlamentarischen Kontrolle entrückte Allerhöchste Kommandogewalt über die gesamte Wehrmacht, die vor [7v] allem deren Einheit gewährleistet, klar und deutlich von der ministerieller Verantwortung und damit der Kontrolle zweier Parlamente unterworfenen Militärverwaltung geschieden wird; so daß eine Trennung der Ar-

\footnotetext{
${ }^{63}$ volle [handschriftlich].
}

mee in je ein die Landwehr in sich aufnehmendes österreichisches und ungarisches Heer auf das Gebiet der Militärverwaltung eingeschränkt bleiben kann, während die gesetzlich abzugrenzende und entsprechend auszubauende Allerhöchste Kommandogewalt in der alleinigen Hand SEINER MAJESTÄT als des gemeinsamen Monarchen aller Länder der Monarchie und des gemeinsamen Kriegsherrn aller Teile der Wehrmacht verbleibt und die beiden administrativ getrennten, zu zwei selbständigen Verwaltungskörpern - nicht aber Kommandoeinheiten - ausgebauten Heere zu einer höheren, zu der in operativer Hinsicht allein maßgebenden strategisch ${ }^{64}$-taktischen Einheit verbindet; wobei erhöhte Garantien für das einheitliche Zusammenwirken der beiden Heere auch auf administrativem Gebiete zu schaffen sind. ${ }^{65}$

Eine auf diesem Prinzipe aufgebaute Reform kann unter grundsätzlicher Aufrechterhaltung des bestehenden staatsrechtlichen Verhältnisses zwischen beiden Staaten der Monarchie, des seit einem halben Jahrhundert bewährten Dualismus und unter möglichster Schonung der geltenden Gesetze insbesondere der beiden Ausgleichsgesetze, durchgeführt werden. Lediglich ihre das Kriegswesen betreffenden Bestimmungen müßten entsprechende Modifikationen erfahren. 6667 [8r, 4 . Einlagebogen]

\footnotetext{
${ }^{64}$ operativ [handschriftlich].

${ }^{65}$ Hier möchte ich an passender Stelle in geeigneter Form das Wesen der erlösenden Formel aussprechen, welches ja darin besteht daß, die Einheitlichkeit besser gewährleistet wird, wie die bisherige sehr fadenscheinige Gemeinsamkeit. [handschriftlich].

${ }_{66}^{6}$ Den Zwecken der Lösung dieses Problems dient der zuliegende ${ }_{\Perp}$ Entwurf des Militärgesetzes“ [handschriftlich].

${ }^{67}$ Pro Konzipient: Hier wäre nur das einzufügen was auf Seite 4 des Einlagebogens 6 unter dem Marginale „Militärgesetz" und das was auf Seite 3 des Einlagebogens 7 unter "Junctim" gesagt ist. - Natürlich entsprechend umstilisieren. So ist Abschnitt I geschlossen, die Folgerung am Schlusse klar ausgedrückt $u$.
} 
II.

[Trennung von Kommandogewalt und Militärverwaltung.] Der Gedanke, die Kommandogewalt von der Militärverwaltung zu sondern, tritt gleichzeitig mit der Verwirklichung des Konstitutionalismus auf und entspringt dem Bedürfnis, ${ }^{68}$ die Führung der Kommandoangelegenheiten ihrer absolute Einheit und rasche Entscheidung erfordernden Natur entsprechend aus dem schwerfälligen System des Konstitutionalismus auszuscheiden, das seinem Wesen nach auf den Ausgleich einander widerstrebender Interessen und Willensrichtungen gerichtet ist. Besonders in Bundesverhältnissen pflegt man das Kommando von der Verwaltung zu scheiden. Auch in der Geschichte unserer Wehrmacht sind wiederholt Ansätze zu einer solchen Entwicklung zu konstatieren. Zweifellos wirkte dieser Gedanke auch in den beiden Ausgleichsgesetzen, sowie er ja auch in unseren beiden Landwehrgesetzen zum Ausdruck kommt. Allein zu einer klaren, konsequenten Durchführung des Prinzips ist es bei der Organisation der Wehrmacht nicht gekommen. Die ungenügende Scheidung von Militärverwaltung und Kommandogewalt macht sich vor allem in der Organisation der militärischen Zentralstellen, insbesondere in der des gemeinsamen Kriegsministeriums geltend. Dem gemeinsamen Kriegsminister obliegt die Militärverwaltung hinsichtlich der gemeinsamen Teile der Wehrmacht. In dieser Richtung steht er unter der in den beiden Ausgleichsgesetzen prinzipiell statuierten, mangels eines entsprechenden Durchführungsgesetzes allerdings nicht praktikablen Ministerver-

Abschnitt II beschäftigt sich ausschließlich mit der Begründung des Milit.Gesetzes. [handschriftlich].

${ }^{68}$ Pro Konzi. Bei der betreffenden Stelle die entsprechenden $\S \S$ des Milit.Gesetzes anweisen. Der Aufbau ist jetzt anders wir sagten schon, es muß ein Milt. Gesetz geschaffen werden $u$. legen es schon bei, daher [unleserlich]. Dennoch wird sich auch in diesem Abschnitt manches kürzen $\mathrm{u}$ stilistisch ändern lassen [handschriftlich]. antwortung. Allein tatsächlich fungiert der Kriegsminister auch als Hilfsorgan des Monarchen, in allen Angelegenheiten, die sich als Leitung, Führung und innere Organisation - der gemeinsamen Teile der Wehrmacht - darstellen und $\left[8^{\mathrm{v}}\right]$ verfassungsmäßig jeder ministeriellen Verantwortung entzogen sind. Mangels einer auch nur annähernd deutlichen gesetzlichen Grenze zwischen Militärverwaltung und Kommandogewalt, angesichts der außerordentlichen vagen Bestimmung durch die Formel Leitung, Führung und innere Organisation, ist die Frage, wo eigentlich die Verantwortung des Ministers beginnt, und seine von der eines verantwortlichen Ministers gänzlich verschiedenen Stellung eines zu unbedingtem Gehorsam verpflichteten Hilfsorgan der Allerhöchsten Kommandogewalt aufhört, gänzlich im Ungewissen. Überschreitungen nach beiden Richtungen sind daher kaum zu vermeiden. Dazu kommt, daß auch von einem rein verwaltungstechnischen Standpunkt zwischen den Agenden der Kommandogewalt und jenen der Militärverwaltung ein nicht unerheblicher Unterschied besteht. Die Militärseelsorge, die Militär - SanitätsJustiz - Bau - Verkehrs - Schul- Verwaltung muß sich in bureaukratischen Formen abspielen, denen sich die eigentlichen Kommandoagenden ihrer Natur nach entziehen.

Eine gesetzliche Abgrenzung der inhaltlich genau zu umschreibenden Kommandogewalt ist daher ${ }^{69}$ erforderlich.

Allerdings ist es nicht leicht, die Agenden der Kommandogewalt inhaltlich präzise zu umschreiben. Nicht leicht auch die Beantwortung der weiteren Frage, ob es sich empfiehlt, die gesetzliche Scheidung von Kommandogewalt und Militärverwaltung organisatorisch, das heißt, in einer mit den Agenden der Kommandogewalt betrauten, von den mit der Militärverwaltung befaßten Zentralstelle prinzipiell geschiedenen Behörde zum Ausdruck kommen zu las-

${ }^{69}$ unbedingt [handschriftlich]. 
sen, sowie dies [9r] etwa in Preußen mit dem Militärkabinett versucht wurde.

[Organisation der Kommandogewalt.] Was zunächst die Organisation der Kommandogewalt betrifft, so läge es nahe, zu ihrer Ausübung als Hilfsorgan des Allerhöchsten Armeeoberkommandanten auch in Friedenszeiten ein Armeeoberkommando aufzustellen, das neben dem verantwortlichen gemeinsamen Kriegsminister $\mathrm{zu}$ fungieren hätte. Auf ein gemeinsamen Kriegsministerium könnte nämlich nicht verzichtet werden, auch wenn die Aufteilung des gemeinsamen Heeres auf zwei Heere Österreichs und Ungarns und somit der hiefür in Betracht kommenden Militärverwaltung auf zwei nationale Kriegsministerien erfolgt. Denn die Verwaltung der Kriegsmarine widerstrebt der für das Heer durchführbaren administrativen Trennung, schon wegen des Außendienstes der k.u.k. Flotte, von der jedes einzelne Kriegsschiff die Gesamtmonarchie repräsentieren muß, so daß eine Spaltung dieser gemeinsamen Flotte in eine österreichische und eine ungarische auch mit Rücksicht auf ihren tatsächlichen Umfang undurchführbar ist. Es muß daher die Marineverwaltung auch in Hinkunft bei einem gemeinsamen verantwortlichen Ministerium verbleiben, als welches, da es sich nicht empfiehlt auch in diesem Punkte schon jetzt eine Änderung des Ausgleiches eintreten zu lassen und ein gemeinsames Marineministerium eintreten zu lassen, nur das gemeinsame Kriegsministerium in Betracht kommt.

Ein gemeinsamer Kriegsminister ist aber auch deshalb notwendig, weil die Verantwortung für die Verwendung der zur Handhabung der Allerhöchsten Kommandogewalt durch gemeinsame Organe parlamentarisch bewilligten Gelder von einem Minister und Mitglied der gemeinsamen Regierung getragen werden тиß Wenn auch die Aus[9r] ̈̈bung der Allerhöchsten Kommandogewalt durch die Person des Monarchen und seine beiden Staaten, beziehungsweise Heeren gemeinsame Hilfsorgane in meritorischer Hinsicht der ministeriellen Verantwortung und parlamentarischen Kontrolle entzogen werden kann und muß, so ist es doch notwendig, daß für die bezügliche Geldgebahrung eine Verantwortung übernommen und eine parlamentarische Kontrolle insbesondere nach der Richtung sichergestellt werde ${ }^{70}$, daß die bewilligten Gelder tatsächlich nur den vorgeschriebenen Zwecken zugeführt werden. Diese Verantwortung kann aber verfassungsmäßig nur ein Minister, nicht aber der Chef des Generalstabes oder ein von SEINER MAJESTÄT ernannter Armeeoberkommandant übernehmen.

Ein gemeinsamer Kriegsminister ist aber schließlich deshalb unentbehrlich, weil eine gemeinsame Verwaltungsstelle bestehen muß, unter deren verfassungsmäßiger Mitwirkung die möglichste Einheitlichkeit der beiden Militärverwaltungen garantiert wird. Zur Herstellung der für das harmonische Zusammenwirken beider Heere notwendigen gegenseitigen Anpassung der beiden Militärverwaltungen ist - eben weil es sich hier um ein Gebiet verantwortlicher Militärverwaltung handelt - als gemeinsames Organ nur ein gemeinsamer Kriegsminister, nicht etwa ein Organ der Kommandogewalt geeignet.

Ist aber der gemeinsame Kriegsminister unvermeidlich, dann würde die Errichtung eines organisatorisch selbständigen Armeeoberkommandos als besonderer Behörde neben dem gemeinsamen Kriegsminister eine außerordentliche Komplikation des obersten Kriegswesens bedeuten. Nicht weniger als vier Zentralstellen hätten sich in diese Leitung zu teilen: das Armeeoberkommando, der gemeinsame, Kriegsminister der österreichische und der ungarische Kriegsminister. [10r , 5. Einlagebogen]

Eine solche Behördenhäufung bedeutete geradezu die Gefahr einer Lahmlegung von Militärverwaltung und Kommandoführung. Auch ist zu bedenken, daß gegen die Errichtung eines Armeeoberkommandos als einer außen hin wirksamen selbständigen Behörde

\footnotetext{
${ }^{70} \mathrm{n}$ muß [handschriftlich].
} 
dieselben Bedenken geltend gemacht werden könnten, die in der zweiten Hälfte des vorigen Jahrhunderts zweimal zu einer Wiederaufhebung des neben einem Kriegsministerium vorübergehend errichteten Armeeoberkommandos geführt haben. Beidemale wurde es unter der unmittelbaren Wirkung vorrangegangener Feldzüge aufgestellte, beidemale aus konstitutionellen Rücksichten wieder mit dem Kriegsministerium vereinigt. Mit Armeebefehl vom 16. Oktober 1849, näher durchgeführt mit Armeebefehl vom 29. Dezember 1850, wurde bei gleichzeitiger Scheidung der Militärverwaltung von der Allerhöchsten Kommandogewalt für die letztere ein Allerhöchstes Armeeoberkommando, für die erstere das bereits seit Einführung des Konstitutionalismus im Jahre 1848 bestehende verantwortliche Kriegsministerium bestimmt. Nach Aufhebung der Verfassung wurden zwar im Jahre 1853 die Agenden des Kriegsministers dem Armeeoberkommando überwiesen, aber sofort bei Wiederaufrichtung der Verfassung wandelte sich das Armeeoberkommando im Jahre 1860 in ein verantwortliches Kriegsministerium um. Nach dem unglücklichen Kriege des Jahres 1866 wurde wieder neben dem Kriegsministerium mit Allerhöchster Entschließung vom 15. September 1866 ein mit den Angelegenheiten der Kommandogewalt betrautes Armeeoberkommando aufgestellt, jedoch schon von vorneherein nur [10v] bis zur Erfüllung einer bestimmten Aufgabe der Reorganisation der Armee. Aber schon im Januar des Jahres 1868 wurde unter Berufung auf die neue Verfassung im Dezember 1867 in der offiziösen WIENER Abendpost bekanntgegeben, daß mit Allerhöchster Entschließung vom 15. Januar 1868 die Geschäfte des Armeeoberkommandos an das Reichskriegsministerium übertragen worden seien.

Es empfiehlt sich daher auch heute, die während des jetzigen Krieges zum größten Teile durch das Armeeoberkommando geführten Agenden der Allerhöchsten Kommandogewalt nach erfolgter Demobilisierung wieder im gemeinsamen Kriegsministerium bearbeiten zu lassen, mit der Änderung: daß die zur Allerhöchsten Kommandogewalt gehörigen und daher außer- halb der ministeriellen Verantwortung und parlamentarischen Kontrolle stehenden Angelegenheiten gesetzlich festgelegt und solcherart klar und deutlich von der durch die beiden nationalen Kriegsminister und durch den gemeinsamen Kriegsminister zu führenden verantwortlichen und parlamentarischen Kontrolle unterworfenen Militärverwaltung geschieden werden. Hinsichtlich der Agenden der Kommandogewalt wäre das gemeinsame Kriegsministerium nur im übertragenen Wirkungskreise, als Organ des Allerhöchsten Oberbefehls, tätig. Nach dieser Richtung hätte es lediglich die vom Chef des Generalstabes zu erteilenden Direktiven auszuführen. Der Chef des Generalstabes hätte als unmittelbares Hilfsorgan des Allerhöchsten Oberbefehles neben dem gemeinsamen Kriegsminister, diesem koordiniert, Immediatvortrag bei SEINER MAJESTÄT. [11 ${ }^{\mathrm{r}}$ ]

[Umfang der Kommandogewalt.] Die Einheit der gesamten Wehrmacht soll aber nicht nur dadurch sichergestellt werden, daß die Allerhöchste Kommandogewalt durch entsprechende gesetzliche Maßnahmen klar und deutlich der ministeriellen Verantwortung und daher den nach verschiedenen Richtungen orientierten Einflüssen zweier Parlamente entrückt wird, sondern auch dadurch, daß in den Kreis der Kommandogewalt alle in die Einheit der Wehrmacht verbürgenden Wirkungsmöglichkeiten aufgenommen werden.

So vor allem die Organisation der Wehrmacht einschließlich der Ernennung und Einteilung der Offiziere, insbesondere auch die Regelung der Dienst- und Kommandosprache. Die Einteilung und Ernennung der Offiziere ist schon darum als Reservatrecht anzusprechen, weil das Offizierskorps, dieses Rückgrat der Armee, in allen wesentlichen Belangen ohne jede fremde Ingerenz dem Allerhöchsten Oberbefehl zur Verfügung bleiben muß. Daß die Regelung der Dienst- und Kommandosprache nur durch einen aus der unverantwortlichen Allerhöchsten Kommandogewalt fließenden Willensakt erfol- 
gen kann, muß aus dem Gesetze unzweideutig hervorgehen.

Weil es für die Einheit der Wehrmacht unbedingt erforderlich ist, daß für alle Teile der Landarmee, insbesondere für die beiden Heere Österreichs und Ungarns nur ${ }^{71}$ ein gemeinsamer Generalstab,72 nur gemeinsame Spezialstäbe (Artillerie- Genie-stab etc.) und gewisse gemeinsame Anstalten für die Ausbildung und für besondere Dienstzweige bestehen, und da nicht mit Sicherheit vorausgesehen werden kann, welche Stäbe und Anstalten hiefür in Betracht kommen, muß der Kommandogewalt die Bestimmung der zu ihrer Handhabung notwendigen und sohin gemeinsamen Organe vorbehalten bleiben. ${ }^{73}\left[11^{\mathrm{v}}\right]$

Zur Organisation der Wehrmacht gehört aber auch die Erlassung genereller Normen für den Dienstbetrieb aller Teile der Wehrmacht, somit aller Reglements und Dienstbücher; daher auch die Disziplinarund Ehrenratsvorschriften. Da die interne Militärverwaltung - soferne nicht Pflichten und Rechte außenstehender, nicht oder noch nicht zum militärischen Organismus gehöriger Personen in Betracht kommen ${ }^{74}$ nach wie vor auf solchen Allerhöchst sanktionierten Reglements und Dienstbüchern beruhen $т и \beta$, wird dieser Teil der Militärverwaltung kraft der einheitlichen Quelle der maßgebenden generellen Normen auch nach administrativer Trennung für das österreichische wie für das ungarische Heer jeden wünschenswerten Grad von Einheitlichkeit behalten.

Was dagegen die auf Grund von Gesetzen und gesetzlich delegierten Verordnungen durchzuführende Verwaltung beider nationaler Kriegs-

\footnotetext{
${ }^{71}$ eine gemeinsame Generalität [handschriftlich].

${ }^{72}$ Pro Konzp: gemeins. Generalst bleibt ungefährl habe Gründe dafür. [handschriftlich].

${ }^{73}$ Abschwächen, man könnte sonst vermuten daß wir uns ins Unendliche erweitern möchten [handschriftlich].

${ }^{74}$ muß [handschriftlich verschoben].
}

ministerien betrifft, - und diese kommt insoweit in Betracht als es sich um die Militärjustizpflege und um für die Militärverwaltung erforderlichen Pflichten und Rechte von nicht zum Militär gehörigen Personen handelt, - so mu $\beta^{75}$ gesetzlich vorgesehen werden, daß die Kriegsminister der beiden Staaten bei der Vorbereitung der in ihr Ressort fallenden Gesetze, bei Erlassung von Verordnungen auf Grund dieser Gesetze, und bei allen prinzipiellen Entscheidungen und Verfügungen das Einverständnis mit dem gemeinsamen Kriegsminister herzustellen haben. Die hier vorgeschlagene Verknüpfung der gemeinsamen Militärverwaltung mit der Sonderverwaltung jedes der beiden Staaten findet ihr Vorbild in einem durch die Rechtsordnung der beiden Staaten bereits geschaffenen und entwickelten Rechtsinstitute sowie es insbesondere in den Vollzugsklauseln der beiden Wehrgesetze normiert ist. [12r, 6. Einlagebogen]

Hinsichtlich der militärischen Gesetze wäre eventuell in Erwägung zu ziehen, ob ihnen nicht allen die gleiche Qualifikation von notwendigerweise paktierten, hinsichtlich ihre Inhaltes zwischen beiden Staaten zu vereinbarenden Normen in der gleichen Weise zuerkannt werden sollten, wie dies gegenwärtig nur hinsichtlich des Wehrgesetzes und zwar unzweideutig nur im österreichischen Ausgleichsgesetze geschieht. ${ }^{76}$

Zur Allerhöchsten Kommandogewalt gehören muß auch die generelle Festsetzung der Bewaffnung und Ausrüstung, während die administrative Beschaffung und Verwaltung des Materials den Kriegsministerien der beiden Staaten überlassen bleiben.

Zur Kommandogewalt muß ferner gehören die Ausbildung der Truppen und Stäbe, die Vorbereitung und Durchführung von Truppenübungen aller Art insbesondere auch von gemeinsamen Truppenübungen der Heere beider Staaten

\footnotetext{
${ }^{75}$ wird [handschriftlich].

${ }^{76}$ Könnte auch wegbleiben [handschriftlich].
} 
und die Inspizierung aller Teile der Wehrmacht durch Generalinspektoren, die als gemeinsame Organe der Allerhöchsten Kommandogewalt unterstellt sind. ${ }^{77}$

Als zur Kommandogewalt muß ferner anerkannt werden: Die Verwendung aller Teile der Wehrmacht und deren Dislokation, worunter Bestimmung der Standorte zu verstehen ist. Der Träger der Allerhöchsten Kommandogewalt muß das Recht haben, alle Teile der Wehrmacht im Frieden wie im Kriege überall zu dislozieren und zu verwenden, wo es das Interesse der Monarchie nach seinem Ermessen erfordert. Die Verwendung jedes Teils der Wehrmacht innerhalb der Gesamtmonarchie ebenso wie außerhalb ihrer oder der Grenzen eines der beiden Staaten, beziehungsweise Bosniens und der Hercegovina durch SEINE MAJESTÄT ergibt sich aus der hinsichtlich der $\left[12^{v}\right]$ gesamtenWehrmacht übertragenen, hinsichtlich der beiden Heere gemeinsam auszuübenden Kommandogewalt. Allfällige Einschränkungen dieses Dislokations- und Verwendungsrechtes in territorialer Hinsicht wie sie derzeit für die ungarische Landwehr bestehen, kommen für keines der beiden neu zu schaffenden Heere in Betracht, da dieses als Rechtsnachfolger den bisher gemeinsamen Heeres anzusehen sind, für das keine derartige Beschränkung bestanden hat.

Als in die Kompetenz des Allerhöchsten Oberbefehl fallend sind schließlich alle die Wehrmacht betreffenden operativen Angelegenheiten anzuerkennen, insbesondere alle Verfügungen über die Wehrmacht während des Krieges, die Anordnung einer teilweisen oder vollen Ergänzung auf den Kriegsstand, die Entlassung zur Wiederannahme des Friedensstandes, die Mobilisierung und Demobilisierung und das Befestigungswesen. Die nähere Durchführung wäre

${ }^{77}$ Ich will aber die Gel Insp. - gleich wie jetzt - als Hilfsorgane dem KM unterstellt haben. [handschriftlich]. auf diesen Gebieten den beiden nationalen Kriegsministern $\mathrm{zu}$ überlassen, die hier als Hilfsorgane der Kommandogewalt aufträten. Nur die bauliche Durchführung der Befestigungsarbeiten hätte als eine Verwaltungsagende zu gelten.

[Die Militärverwaltung.] Alle die Heere der beiden Staaten betreffenden Agenden, soferne die nicht der Kommandogewalt oder dem Wirkungskreis des gemeinsamen Kriegsministers vorbehalten sind, gehören in die Kompetenz des k.k. österreichischen, beziehungsweise des k.u. Kriegsministers. Diesen obliegt auch für den Bereich ihres Staates die politisch-administrative Tätigkeit hinsichtlich der gemeinsamen Teile der Wehrmacht.

[Die Kosten.] Die Kosten der durch die beiden Kriegsminister geführten Militärverwaltungen würden in Hinkunft nicht mehr gemeinsam sein, sondern den betreffenden Staat allein be[13r]lasten, so daß Österreich für das österreichische Heer, Ungarn für das ungarische Heer zu sorgen hätte. Dagegen müßten die Kosten der Ausübung der Kommandogewalt und der vom gemeinsamen Kriegsminister geführten Verwaltung, insbesondere der k.u.k. Kriegsmarine, im Sinne der beiden Ausgleichsgesetze gemeinsam bleiben. ${ }^{78}$ Als gemeinsam müßten auch die Kosten aller Befestigungen anerkannt werden, da sonst der österreichische Staat mit seiner unverhältnismäßig größeren Auslandsgrenze eine mit Rücksicht auf den auch für den andern Staat entscheidenden Wert der österreichischen Grenzbefestigungen zu starke finanzielle Belastung erfahren würde. Gemeinsam müßten auch bleiben die Kosten der Mobilisierung jedes Teiles der bewaffneten Macht und vor allem die Kosten der Kriegsführung. Denn es müsste die Einheit der Kriegsführung gefährden, wenn man beiden Staaten der Monarchie ein geson-

\footnotetext{
${ }^{78}$ Vom b h. Heereskontingent ist nirgends die Rede [handschriftlich].
} 
dertes Interesse an der Höhe der Kriegskosten bei gemeinsamer Kriegsführung einräumen würde. So werden ja auch gegenwärtig die durch die Mobilisierung und Verwendung der Landwehren zu Kriegszwecken entstehenden Kosten vom gemeinsamen Kriegsministerium bestritten.

Mit einer solchen Regelung der Kostenfrage würde die so überaus schwierige und das politische Verhältnis zwischen beiden Staaten immer wieder trübende Festsetzung 79 der Quote für das Beitragsverhältnis zu den gemeinsamen Ausgaben entweder überhaupt überflüssig werden oder doch mit Rücksicht auf die geringe Höhe des quotenmäßig noch zu bedeckenden Betrages jene Bedeutung verlieren. Es ist zu vermuten, daß durch die reinen Zollüberschüsse alle noch verbleibenden Ausgaben oder doch zumindest der größte Teil derselben gedeckt werden könnten.[13v]

Nach der Schlußrechnung für das Jahr 1913 beliefen sich die gesamten gemeinsamen Kosten auf K 912,395,632.01, da hievon durch reine Zollüberschüsse K 227,669,064.43 gedeckt wurden, so verblieben für die Bestreitung durch Quotenbeiträge K 684,726,567.58. Diesen stehen gegenüber als Ausgaben für das gemeinsame Heer: K 640.921.313. Nach Abzug dieser Post würde ein Betrag von kaum 44 Millionen für die quotenmäßige Deckung in Betracht kommen.

[Rechtskontrolle über die Militärverwaltung.] Als eine wichtige und wertvolle Konsequenz der geplanten Reform wäre auch die Tatsache in Rechnung zu ziehen, daß durch eine administrative Trennung des gemeinsamen Heeres und die Aufteilung der Militärverwaltung auf beide Staaten die Möglichkeit einer Rechtskontrolle

\footnotetext{
${ }^{79}$ der quotenmäßigen Aufteilung sämtl. Lieferungen auf beide Staaten so wie der Quote überhaupt - (entsprechend stilisieren). das KM. will zunächst den fortwährenden Streit um die quotenmäßige Aufteilung vermeiden dann käme aber vom Standpunkt beider Staaten die Quotenfrage in Betracht. [handschriftlich].
}

über die Militärverwaltung durch die Gerichtshöfe öffentlichen Rechtes beider Staaten ermöglicht würde, denen die dringend notwendige im Interesse der Heeresverwaltung ebenso wie der Staatsbürger gelegenen Rechtskontrolle eben wegen der Gemeinsamkeit der Militärverwaltung bisher entrückt war.

[Das Militärgesetz.] Was die legislative Durchführung der geplanten Reform betrifft, so wären die hier entwickelten Grundzüge in zwei textlich genauestens übereinstimmenden Gesetzen, in einem österreichischen und einem ungarischen Militärgesetz durchzuführen, für das ein Entwurf als Basis weiterer Verhandlungen angeschlossen wird. Dieses Gesetz hätte ${ }^{80}$ sich als eine authentische Interpretation und weitere Durchführung beziehungsweise Modifikation des staatsrechtlichen Ausgleichs vom Jahre 1867 auf dem Gebiete des Kriegswesens darzustellen. Es hätte, um bezüglich Verfassungsstreitigkeiten hinsichtlich seiner Abänderungsmöglichkeit von vornherein auszuschalten, eine ausdrückliche Bestimmung zu enthalten, die jede einseitige Abänderung dieses Gesetzes durch die Legislative eines der beiden Staaten ausschließt. ${ }^{81}\left[14^{\mathrm{r}}\right.$, 7. Einlagebogen]

Mit diesem Militärgesetz wäre die Aufhebung der beiden Landwehrgesetze und sonstiger durch die Reform bedingte Änderungen der Rechtsordnungen beider Staaten zu verbinden.

1.)

Insbesondere wäre das tirolische Landesgesetz betreffend das Landesverteidigungsinstitut das ja als solches im Gefüge des österreichischen Heeres bestehen bleiben könnte, - der neuen Organisation entsprechend zu modifizieren.

1.) und 2.)

Was speziell die Justizgesetze betrifft, so wären für die gemeinsam verbleibenden Teile der

\footnotetext{
${ }^{80}$ stellt [handschriftlich].

${ }^{81}$ Kommt auf Seite 4 des 3. Einlagebogens [handschriftlich].
} 
Wehrmacht die gegenwärtig bestehenden Normen aufrecht zu erhalten. Für das österreichische, beziehungsweise das ungarische Heer wären Militärstrafgesetz und MilitärStrafprozeßordnung, sowie wie bisher für die beiden Landwehren in Geltung stehen, zu übertragen. Die Angehörigen der beiden Heere, die bei gemeinsamen Institutionen eingeteilt sind, bleiben der Gerichtsbarkeit desjenigen Heeres unterworfen, dem sie angehören.

Schließlich wäre im Zuge der Reform moderne, die Erfahrungen dieses Krieges berücksichtigende Wehrgesetze zu schaffen..$^{82}$

[Organisation der beiden Heere.] Was die auf Grund des $^{83}$ reservierten Organisationsrechtes SEINER MAJESTÄT ${ }^{84}$ durchzuführende Organisation der beiden Heere betrifft, so sei nur so viel bemerk $t^{85},\left[14^{\mathrm{v}}\right]$ daß sie die beiden Landwehren in sich aufzunehmen hätte und daß auf solche Weise an Stelle von drei Heeren nur mehr deren zwei träten. Beide Heere wären mit allen Symbolen und Abzeichen ihres Staates auszustatten, nur bei den gemeinsamen Institutionen hätten gemeinsame Symbole und Abzeichen zu bestehen. Als Dienst- und Kommandosprache wäre in der österreichischen Armee ${ }^{86}$ die deutsche, in der ungarischen die ungarische einzuführen, wobei jedoch in entsprechender Weise die kroatische Sprache im Gefüge des ungarischen Heeres berücksichtigt werden müßte. Im Verkehre vom Korpskommando aufwärts wäre unter allen Umständen die deutsche Sprache vorgeschrieben. Ebenso wäre die deutsche Sprache in

\footnotetext{
${ }^{82}$ Wofür ich nur, nach Maßgabe des Fortschreitens der Verhandlungen über das vorliegende Milit Gesetz, Vorschläge zu erstatten erlauben werde [handschriftlich].

${ }^{83}$ unvorgreiflich dem [handschriftlich].

${ }^{84}$ möchte ich über die [handschriftlich].

85 en [handschriftlich].

${ }^{86}$ Und in den gemeinsam verbleibenden Teilen der Wehrmacht (Kriegsmarine, bh. Heereskontingent) ebenso in den beiden Heeren gemeinsamen Institutionen [maschinschriftlich]
}

allen die Führung berührenden taktischen, operativen und generalsstabs-technischen Angelegenheiten als Dispositionssprache und im amtlichen Verkehr der beiden Heere untereinander und den anderen Teilen der Wehrmacht als Verständigungssprache anzuerkennen. Auch wären wegen Erlernung der deutschen Sprache in den militärischen Bildungsanstalten und bei der Zulassung zur Ausbildung für die Stäbe entsprechende Maßnahmen zu treffen. In allen Teilen der Wehrmacht wäre aber auf die Muttersprache des Mannes, sowie dies schon gegenwärtig der Fall ist, im Interesse der Ausbildung Rücksicht zu nehmen.

[Junctim.] Die gesamte Durchführung der Reform kann selbstverständlich erst in der Zeit nach Abschluß des Krieges in Frage kommen. Dabei muß seitens der gemeinsamen Kriegsverwaltung der Grundsatz aufgestellt werden, daß die SEINER MAJESTÄT reservierte Aufstellung der beiden Heere nur [15r] zugleich mit der Sanktion aller derjenigen Gesetze erfolgen kann, die die Voraussetzung der Reform bilden und alle militärischerseits $\mathrm{zu}$ fordernden Garantien enthalten. ${ }^{8788}$

\footnotetext{
${ }^{87}$ Kommt auf Seite 4 des 3. Einlagebogens als Schluss [handschriftlich].

${ }^{88}$ Entsprechender Schluss - etwa so - Ich bitte EE. diese meine Vorschläge einer geneigten Prüfung zu unterziehen und mir dann Gegenvorschläge ehestens zukommen zu lassen. Ich halte ein festes Einverständnis zwischen den Chefs der beiden Reg. u. dem KM. für dringend nötig um angesichts der fast tägl. wechselnden außen- $u$. innenpolit Situat. nicht in die Lage versetzt zu werden, ohne Richtlinie, ohne Einigung über die Grundsätze auf welchen Reform erfolgen soll bzw ohne volle Klarheit zu haben wie sich die Armeefrage zu entwickeln hätte.

bin auch bereit in mündl. Verhandlungen einzutreten u. stehe EE. jederzeit zu [unleserlich].

diese Note gleichlautend
} 


\section{Entwurf für ein Militärgesetz ${ }^{89}$}

\section{Die Bestimmung der Wehrmacht $\S 1$.}

Die Wehrmacht der österreichisch-ungarischen Monarchie ist in ihrer Gesamtheit wie in jedem ihrer Teile zur Verteidigung des Gebietes sämtlicher unter der Herrschaft Seiner kaiserlichen und königlichen APOSTOLISCHEN MAJESTÄT stehenden Länder gegen äußere Feinde und zur Aufrechterhaltung der Ordnung und Sicherheit im Innern bestimmt.

\section{Die Gliederung der Wehrmacht $§ 2$.}

Die Wehrmacht der österreichisch-ungarischen Monarchie gliedert sich in administrativer Hinsicht in das kaiserlich-königliche österreichische und das königlich ungarische Heer, die kaiserliche und königliche gemeinsame Kriegsmarine und das kaiserlich und königliche bosnischhercegovinische Heereskontingent.

\section{Die Kommandogewalt $\S 3$.}

Die oberste Kommandogewalt über alle Teile der Wehrmacht steht im Frieden und im Kriege im Sinne des Gesetzes vom 21. Dezember 1867, Reichsgesetzblatt Nr. 146 (Gesetzartikel XII vom Jahre 1867) ausschließlich und ungeteilt Seiner kaiserlichen und königlichen APOSTOLISCHEN MAJEST ̈̈T zu.

\section{$\S 4$.}

Zur obersten Kommandogewalt gehören:

1. Die Organisation der Wehrmacht einschließlich der Ernennung und Einteilung der Offiziere; insbesondere auch die Regelung der Dienst- und Kommandosprache, die Bestimmung der zur Handhabung der Kommandogewalt notwendigen allen oder nur einzelnen Teilen der Wehrmacht gemeinsamen Organe (Stäbe und Anstalten), die Erlassung genereller Normen für den Dienstbetrieb aller Teile der Wehrmacht (Reglements, Dienstbücher), die Festsetzung der Bewaffnung, Bekleidung und Ausrüstung.

\footnotetext{
${ }^{89}$ OberKOFleR, RABOFSKY, Kelsen im Kriegseinsatz
} 187-190.
2. Die Ausbildung der Truppen und Stäbe, die Vorbereitung und Durchführung von Truppenübungen aller Art und die Inspizierung aller Teile der Wehrmacht.

3. Die Verwendung aller Teile der Wehrmacht und deren Dislokation, das ist die Bestimmung ihrer Standorte.

4. Alle die Wehrmacht betreffenden operativen Angelegenheiten, insbesondere alle Verfügungen über die Wehrmacht während des Krieges, die teilweise oder volle Ergänzung auf den Kriegsstand sowie die Entlassung zur Wiederannahme des Friedensstandes, die Mobilisierung und Demobilisierung, das Befestigungswesen.

Die Militärverwaltung: der österreichische (ungarische) Kriegsminister $\S 5$.

Zur Durchführung der gesamten Militärverwaltung ist für das österreichische (ungarische) Heer ist ein verantwortlicher kaiserlichköniglich österreichischer (königlich ungarischer) Kriegsminister als Mitglied der österreichischen (ungarischen) Regierung berufen.

Ihm obliegt auch die politisch-administrative Tätigkeit hinsichtlich der gemeinsamen Teile der Wehrmacht für den Bereich der österreichischen Länder (Länder der ungarischen heiligen Krone).

Er hat bei der Vorbereitung der in sein Ressort fallenden Gesetze, bei der Erlassung von Verordnungen auf Grund dieser Gesetze und bei allen prinzipiellen Entscheidungen und Verfügungen das Einverständnis mit dem kaiserlichen und königlichen gemeinsamen Kriegsminister herzustellen.

Der österreichische (ungarische) Kriegsminister fungiert als Organ der Kommandogewalt, sofern ihm von SEINER MAJESTÄT auch die Durchführung von im $\S 4$ bezeichneten Agenden für das österreichische (ungarische) Heer übertragen wird. 


\section{$\S 6$.}

Zu der im §5, Absatz 1, erwähnten Militärverwaltung gehören alle das österreichische (ungarische) Heer betreffenden Angelegenheiten, sofern sie nicht im $\S 4$ der Kommandogewalt SEINER MAJESTÄT vorbehalten sind oder in den im $\S 7$ umschriebenen Wirkungskreis des gemeinsamen Kriegsministers fallen.

\section{Die Militärverwaltung:}

\section{Der gemeinsame Kriegsminister $§ 7$.}

Der gemeinsame Kriegsminister ist für die budgetmäßige Verwendung der zur Ausübung der Kommandogewalt jährlich bewilligten Gelder den Delegationen im Sinne des Gesetzes vom 21. Dezember 1867, R.G.Bl. Nr. 146 (Gesetzartikel XII vom Jahre 1867) verantwortlich.

Er hat in den im $\S 5$, Absatz 3 genannten Angelegenheiten mit den Kriegsministern der beiden Staaten der Monarchie das Einverständnis herzustellen. Im Falle ein Einvernehmen nicht zu erzielen ist, entscheidet SEINE MAJESTÄT.

Hinsichtlich der gemeinsamen Kriegsmarineverbleibt dem gemeinsamen Kriegsminister seine im Gesetze vom 21. Dezember 1867, R.G.Bl. Nr. 146 (Gesetzartikel XII vom Jahre 1867) begründete Kompetenz und Verantwortung.

Dem gemeinsamen Kriegsminister steht hinsichtlich des bosnisch-hercegovinischen Heereskontingents die Militärverwaltung zu.

Als Organ der Kommandogewalt bearbeitet das gemeinsame Kriegsministerium die im $\S 4$ bezeichneten Agenden.

\section{Die Kosten § 8 .}

Die Kosten der Ausübung der Kommandogewalt und der vom gemeinsamen Kriegsminister geführten Militärverwaltung (mit Ausnahme des b.h. Heereskontingentes), ebenso die Kosten aller Befestigungen, der Mobilisierung jedes Teils der Wehrmacht und der Kriegsführung sind gemeinsam.

Dagegen belasten die Kosten der von dem österreichischen (ungarischen) Kriegsminister ge- führten Militärverwaltung ausschließlich den österreichischen (ungarischen) Staat.

\section{Die Aufhebung der Landwehr § 9.}

Das Gesetz vom 5. Juli 1912, R.G.Bl. Nr. 129 über die k.k. Landwehr etc. (der Gesetzartikel XXXI vom Jahre 1912 über die Landwehr) wird außer Kraft gesetzt.

\section{Die Abänderung des Militärgesetzes § 10.}

Eine Abänderung dieses Gesetzes kann nur erfolgen, wenn das ungarische Militärgesetz, Gesetzartikel ....vom Jahre.... (das österreichische Militärgesetz, Gesetz vom....... R.G.Bl. Nr.....) im gleichen Sinne abgeändert wird.

\section{Gutachten 2}

7-seitiges Typoskript mit handschriftlichen Ergänzungen, ÖNB, Nachlass Stöger-Steiner, Cod. Ser. n. 54580;

Unterstreichungen im Original werden übernommen. Die handschriftlichen Ergänzungen, die nicht von Kelsen, vermutlich aber von Stöger-Steiner stammen, wurden als Fußnoten wiedergegeben. Offenkundige Schreibfehler wurden korrigiert.

\section{Text}

Die Aufgaben der Heeresverwaltung bei einer Verfassungsreform von Universitätsprofessor Dr. Hans Kelsen

Die gewaltigen wirtschaftlichen und politischen Umwälzungen, die der Weltkrieg herbeigeführt hat, müssen sich in einem wegen der nationalen und staatsrechtlichen Verhältnisse so komplizierten und empfindlichen Organismus, wie es die österr.-ung. Monarchie ist, ganz besonders fühlbar machen. Darum ist es nur selbstverständlich, daß der Weltkrieg die Grundprobleme der Staatsverfassung in Österreich wie in Ungarn aufgerührt hat. Hier ist es die Frage des Wahlrechtes, dort die nationale Autonomie, die in den Vordergrund des politischen Interesses gerückt ist; in beiden Staaten aber das immer 
stärker vordringende Prinzip der Demokratisierung und Parlamentarisierung.

Aber auch eine Revision des staatsrechtlichen Verhältnisses zwischen beiden Staaten der Monarchie steht bevor, u.zw. vor allem auf dem Gebiete des Militärrechtes, da hier die völlige Durchführung eines paritätischen Dualismus und die bedingungslose Anerkennung der Souveränität beider Staaten der Monarchie im Geiste des Ausgleiches v. 1867 noch aussteht u. von ungarischer Seite, gestützt auf die hohen Verdienste der ung. Truppen und die Erfahrungen dieses Krieges mit allem Nachdrucke gefordert werden wird. Zumal gerade in Ungarn jene Parteien zur Regierung gelangt sind, die seit je her die verfassungsrechtlichen Postulate auf dem Gebiete des Militärrechtes vertreten haben und gerade auf diesem Gebiete einen politischen Erfolg erringen müssen.

In der großen Bewegung der Verfassungsrevision [3] werden daher die Fragen der militärischen Organisation und deren Stellung innerhalb der staatlichen Ordnung eine bedeutende Rolle spielen. An diese Heeresverwaltung wird die große Aufgabe herantreten, bei der Neugestaltung der verfassungsrechtlichen Grundlagen der Wehrmacht entscheidend mitzuwirken. Dabei besteht nicht nur die Gefahr, daß der Heeresverwaltung bei dieser Entscheidung der ihr gebührende Einfluss geschmälert werde, sondern auch, daß die hier maßgebenden militärischen Stellen von der an sie herantretenden Aufgabe unvorbereitet angetroffen werden. Im letzteren Falle ist die unter alle Umständen zu vermeidende Möglichkeit gegeben, daß in dem sich entwickelnden Kampfe widerstreitender Interessen militärischerseits zu großes Gewicht auf die Erhaltung gewisser traditioneller äusserer Formen gelegt wird, deren Opferung für berechtigte nationale Aspirationen bei eingehender Prüfung und kluger Erwägung aller relevanten Umstände ohne ernstliche Verletzung der nicht an der Oberfläche äusserer Formen, sondern tiefer liegenden Lebensnerven der militärischen Interessen möglich wäre. ${ }^{90}$ Wie viel überflüssiger Streit, wie viel gegenseitige Entfremdung hätte in dem Verhältnis der beiden vom Schicksal zusammen gefügten und zusammengehörigen Staaten unserer Monarchie vermieden werden können, wenn man immer nur auf die Sache und nicht so sehr auf die äussere Form gesehen und darum bei einer stolzen und selbstbewussten Nation - die gerade in ihrem nationalem Ehrgeiz umso wirksamer in den Dienst der Großmachtinteressen der Monarchie hätte gestellt werden können - den berechtigten, auf äussere Anerkennung ihrer Souveränität gerichteten Drang nicht durch eine inkonsequente Politik der halben und kleinen Mittel zu hemmen versucht hätte!

Gerade auf dem Gebiete des MilitärVerfassungsrechtes, wo dieser Kampf unmittelbar bevorsteht, ist die [4] geschilderte Gefahr doppelt so groß. Darum gilt es nach dieser Richtung eine Formel zu finden, die sowohl dem in diesem Kriege gesteigerten Selbstgefühle der Nationen ${ }^{91}$ und insbesondere der ung. Nation, als auch dem unverzichtbaren Anspruch auf materielle Einheit der Wehrmacht entspricht.

Dies ist aber nicht ohne gründliche Vorbereitung aller hier in Betracht kommender Fragenmöglich. Zur Bearbeitung dieser Materien ist daher die Errichtung einer Stelle unumgänglich notwendig, die den ganzen Komplex der hier in Betracht kommenden Probleme zu untersuchen und entsprechende Reformentwürfe auszuarbeiten hat. Diese Stelle muss - wo immer sie errichtet wird $^{92}$ - in innigstem Kontakte mit allen maßgebenden militärischen Behörden, muss innerhalb des militärischen Organismus selber stehen.

Die Aufgabe dieser Stelle wäre nicht nur die verfassungsrechtliche Stellung der Wehrmacht

\footnotetext{
${ }^{90}$ Richtig, darüber ist man sich auch schon klar. 91 ?

${ }^{92}$ Diese Stelle kann nur beim KM sein.
} 
im Verhältnis zu den beiden Staaten der Monarchie - also im Hinblick auf das bestehende dualistische System - zu revidieren, sondern auch die sehr bedeutenden verfassungsrechtlichen und verwaltungsrechtlichen Fragen zu untersuchen, die sich gerade während dieses Krieges auf dem Gebiete der inneren Organisation der Wehrmacht ergeben haben. So ist die Trennung von Kommando und Militärverwaltung, das Verhältnis des AOK. zum Kriegsministerium, die Behebung der unverträglichen Komplikation, die sich durch das schwerfällige Zusammenwirken dreier militärischer Ministerien: des Kriegsministeriums und der beiden Ministerien für Landesverteidigung ergibt, die Abgrenzung der gesetzgebenden und der Verordnungsgewalt, die Rechtskontrolle auf dem Gebiete der Militärverwaltung und anderes mehr. Insbesondere aber hätte die zu errichtende Stelle auch [5] das Problem des Wehrsystems u.zw. insoferne zu bearbeiten, als das Wehrsystem mit der Verfassung des Staates organisch zusammenhängt. Gerade nach dieser Richtung wird die mit immer stärkerer Gewalt vordringende demokratische Bewegung nicht ohne einschneidende Rückwirkung auf die Wehrverfassung bleiben können. Darum müssen an einer militärischen Stelle alle einschlägigen Fragen rechtzeitig durchgearbeitet werden, damit die an Ah. Stelle zur Beratung herangezogenen Militärs aus dem Stande der ihnen unmittelbar unterstehenden Organisation die erforderlichen Informationen holen können.

Im engsten Zusammenhange mit der unaufhaltsamen Entwicklung der Demokratie steht auch die zunehmende Macht gewisser demokratischer Schlagworte, zu deren charakteristischesten der „Kampf gegen den Militarismus“ gehört. $\mathrm{Daß}$ dieses zum großen Teil unberechtigte Schlagwort eine nicht zu unterschätzende Bedeutung im politischen Leben bekommen hat und nach diesem Krieg zweifellos eine noch viel größere Bedeutung erlangen wird, darin liegt die Schuld zum Teil auf militärischer Seite, da man hier die große Öffentlichkeit über die Organisation des militärischen Apparates und über sein tatsächliches Funktionieren gänzlich uninformiert ließ, während doch eine entsprechende Aufklärung der Bevölkerung über Verhältnisse, die keinerlei Licht zu scheuen hatten, viel Mißverständnisse hätte vermeiden können. Hätte der Rekrut bei seinem Eintritt in die militärische Organisation nicht das Gefühl gehabt, in ein ihm gänzlich unbekanntes und nach allgemeiner Meinung rechtloses Gebiet zu geraten, wäre das Schlagwort vom Militarismus nicht $\mathrm{zu}$ jener bedrohlichen Macht geworden, die es heute [6] darstellt.

Bei der Reform der verfassungsrechtlichen Grundlagen der Wehrmacht wird daher der mit den Vorbereitungen betrauten Stelle die wichtige Aufgabe zufallen, die militärische Organisation aus ihrer isolierten Stellung innerhalb des gesamtstaatlichen Organismus zu befreien, den natürlichen Zusammenhang herzustellen, der zwischen der militärischen Verwaltung und allen anderen Zweigen der staatlichen Tätigkeit bestehen muß und für Einrichtungen Sorge zu tragen, die Gewähr leisten, daß der allgemeine Staatsgeist auch den militärischen Verwaltungskörper durchdringe, ${ }^{93}$ der nicht mehr als Staat im Staate, sondern als ein Glied des Staates von jedermann empfunden werden muss, der als Soldat das Bewusstsein behalten kann, Staatsbürger zu sein. Daß die Armee das Volk in Waffen sei, wird in Zukunft auch in der Organisation der Armee zum Ausdruck kommen müssen. Dann erst wird das für die Verteidigung des Staates gefährliche Schlagwort vom „Militarismus" entkräftet sein. ${ }^{94}$

Ebenso wie die vorauszusehende Demokratisierung wird eine früher oder später unvermeidliche nationale Föderalisierung jedes der beiden Staaten auf die verfassungs- und verwaltungs-

\footnotetext{
${ }^{93}$ Jawohl

${ }^{94}$ Allgem. Wehrpflicht auf breitester Basis, damit nähert man sich also dem Milizsystem
} 
rechtliche Stellung der Wehrmacht eine nicht unbeträchtliche Wirkung ausüben. In noch höherem Maße müsste aber eine eventuelle Revision des Dualismus, etwa die Einführung des Trialismus oder gar eine noch radikalere Änderung der Struktur der Monarchie für die Verfassung der Wehrmacht von Bedeutung werden. Auch nach dieser Richtung müssen daher zwecks Sicherung der militärischen Interessen entsprechende Vorbereitungen getroffen werden.

Für die in Vorschlag gebrachte Stelle zur Be[7]arbeitung aller die Wehrmacht betreffenden verfassungs- und verwaltungsrechtlichen Fragen erwächst auch die wichtige Aufgabe, geeignete Vorschläge zur Verbreitung der Kenntnis der militärischen Organisation auszuarbeiten. Hier ist vor allem die Frage zu prüfen, wie das in das Leben des einzelnen so tief einschneidende Heerwesen und Militärrecht zum Gegenstand des Unterrichtes insbesondere an den Hochschulen gemacht werden kann.

Schließlich sei hervorgehoben, daß von der zu organisierenden Stelle nicht nur die militärverfassungs- und verwaltungsrechtlichen, sondern auch die wichtigen völkerrechtlichen Fragen zu bearbeiten wären, die sich in diesem Kriege speziell auf militärischem Gebiete ergeben haben. Wenn auch die bevorstehende Reform des Land- und Seekriegsrechtes hinsichtlich der internationalen Beratung und vertragsmäßigen Perfektuierung zweifellos in die Kompetenz des Ministerium des Äussern fällt, so muss doch die vom spezifisch militärischen Standpunkt notwendige Bearbeitung des einschlägigen Materials, die Verwertung der auf diesem Gebiete nur von militärischer Seite gemachten Erfahrungen und die Formulierung der vom Standpunkte der militärischen Interessen gebotenen Forderungen von einer militärischen, dabei allerdings juristisch entsprechend qualifizierten Stelle erfolgen, die sich ihrerseits mit dem Ministerium des Äussern wird ins Einvernehmen zu setzen haben. ${ }^{95}$

\section{Korrespondenz:}

DDr. Christoph SCHMETTERER

Universität Wien,

Rechtswissenschaftliche Fakultät

Institut für Rechts- und Verfassungsgeschichte

Abteilung KRGÖ

Strohgasse 45/2d, 1030 Wien

christoph.schmetterer@univie.ac.at

\section{Literatur:}

Johann Christoph Allmayer-Beck, Die Bewaffnete Macht in Staat und Gesellschaft, in: Adam WANDRUSZKA, Peter URBAnITSCH (Hgg.), Die Habsburgermonarchie 1848-1918, Bd. V: Die Bewaffnete Macht (Wien 1987) 1-141.

Detlef BALD, Die Bundeswehr. Eine kritische Geschichte 1955-2005 (München 2005).

Eduard BerNATZIK (Hg.), Die österreichischen Verfassungsgesetze mit Erläuterungen (Wien 21911).

Jürgen BusCH, Hans Kelsen im Ersten Weltkrieg. Achsenzeit einer Weltkarriere, in: Robert WALTER, Werner OgRIs, Thomas OleCHOWSKI (Hgg.), Hans Kelsen. Leben - Werk - Wirksamkeit (=Schriftenreihe des Hans Kelsen-Instituts 32, Wien 2009) 57-80

Christian FRECH, Die Armeefrage in ÖsterreichUngarn nach dem Ausgleich 1867 bis 1914 (phil. Dipl.Arb., Univ. Wien 1988).

Hans von FRISCH, Die Verantwortlichkeit der Monarchen und höchsten Magistrate (Berlin 1904).

Lothar HÖBELT, The Delegations. Preliminary Sketch of a Semi-Parliamentary Institution, in: Parlaments, Estates and Representations 6 (1986) 149154.

Ernst Rudolf HuBER, Deutsche Verfassungsgeschichte seit 1789, Bd. III: Bismarck und das Reich (Stuttgart 1963).

${ }^{95}$ Für derartige Vorschläge ist in erster Linie das A.O.K. berufen, welches in diesem Sinne anzugehen wäre. Das KM. hat jedenfalls mitzusprechen, daher gemeinsame Arbeit nötig. Das Entsprechende wäre ehestens zu veranlassen. 
Hans KELSEN, Zur Reform der verfassungsrechtlichen Grundlagen der Wehrmacht Österreich-Ungarns, in: Zeitschrift für Militärrecht 1 (1917) 8-23 (= HKW III, 623).

Gustav Kolmer, Parlament und Verfassung in Österreich, Bd. 8: 1900-1914 (Wien 1914).

Friedrich MARSCHALL VON BIEBERSTEIN, Verantwortlichkeit und Gegenzeichnung bei Anordnungen des Obersten Kriegsherrn. Studie zum deutschen Staatsrecht (Berlin 1911).

Rudolf Aladár MÉTALL, Hans Kelsen: Leben und Werk (Wien 1969).

Karin OLECHOWSKI-HRDLICKA, Die gemeinsamen Angelegenheiten der Österreichisch-Ungarischen Monarchie. Vorgeschichte - Ausgleich 1867 Staatsrechtliche Kontroversen (= Rechtshistorische Reihe 232, Frankfurt am Main 2001).

Gerhard OBerKofler, Eduard RABOFSKy, Hans Kelsen im Kriegseinsatz der k. u. k. Wehrmacht (= Rechtshistorische Reihe 58, Frankfurt am Main 1988).

Tibor PAPP, Die königlich ungarische Landwehr (Honved) 1868 bis 1914, in: Adam WANDRUSZKA, Peter URBANITSCH (Hgg.), Die Habsburgermonarchie 1848-1918, Bd. V: Die Bewaffnete Macht (Wien 1987) 634-686.

Peter PERNTHALER, Der Rechtsstaat und sein Heer. Strukturelemente der österreichischen Wehrverfassung mit vergleichenden Hinweisen auf die Wehrordnungen der Schweiz und der Bundesrepublik Deutschland (= Rechts- und Staatswissenschaften 19, Wien 1964) 19.

László PÉTER, Die Verfassungsentwicklung in Ungarn, in: Peter Urbanitsch, Helmut RuMPLeR (Hgg.), Die Habsburgermonarchie 1848-1918, Bd. VII: Verfassung und Parlamentarismus, Teilbd. 1: Verfassungsrecht, Verfassungswirklichkeit, zentrale Repräsentativkörperschaften (Wien 2000) 239-540.

Christoph SCHMETTERER, Der Kaiser von Österreich als Oberster Kriegsherr, in: Journal on European History of Law 4/1 (2013) 10-18
Ferdinand SCHMID, Das Heeresrecht der österreichisch-ungarischen Monarchie (Wien 1903).

Ferdinand SCHMID, Heerwesen, in: Ernst MISCHLER, Josef UlbRICH (Hgg.), Österreichisches Staatswörterbuch. Handbuch des gesamten öffentlichen österreichischen Rechts, Bd. 2 (Wien 21906) 736-791.

Antonio SCHMIDT-BRENTANO, Stöger-Steiner von Steinstätten Rudolf Frh.. in: ÖBL, Bd. 13 (Wien 2010) 291.

Éva SOMOGYI, Die Delegation als Verbindungsinsitution zwischen Cis- und Transleithanien, in: Helmut RuMPLER, Peter URBANITSCH (Hgg.), Die Habsburgermonarchie 1848-1918, Bd. VII: Verfassung Parlamentarismus, 1. Teilbd.: Verfassungsrecht, Verfassungswirklichkeit, zentrale Repräsentativkörperschaften (Wien 2000) 1107-1176.

Peter STEINER, Sr. Majestät wirkl. Geheimer Rat k.k. Generaloberst Rudolf Frhr. Stöger-Steiner v. Steinstätten Österreich-Ungarns letzter Kriegsminister (phil. Diss., Univ. Innsbruck 1989).

Friedrich TEZNER, Der Kaiser (Wien 1909).

Josef UlBRICH, Lehrbuch des österreichischen Staatsrechts (Berlin 1883).

Walter WAGNER, Geschichte des k.k. Kriegsministeriums, 2 Bde. (=Studien zur Geschichte der österreichisch-ungarischen Monarchie 5, 10, GrazWien-Köln 1966, 1971)

Walter WAGNER, Die k.(u.)k. Armee. Gliederung und Aufgabenstellung, in: Adam WANDRUSZKA, Peter URBANITSCH (Hgg.), Die Habsburgermonarchie 1848-1918, Bd. V: Die Bewaffnete Macht (Wien 1987) 142-633.

Rainer WOHLFEIL, Hans DOLLINGER, Die deutsche Reichswehr. Bilder, Dokumente, Texte. Zur Geschichte des Hunderttausend-Mann-Heeres 19191933 (Frankfurt am Main 1972)

Ivan ZoLGER, Die staatsrechtlichen Grundlagen der Wehrmacht Österreich-Ungarns, in: Österreichische Zeitschrift für öffentliches Recht 2 (1915-16) 525-615. 